\title{
On-board Spacecraft Relative Pose Estimation with High-Order Extended Kalman Filter
}

\author{
Francesco Cavenago $^{\mathrm{a}, *}$, Pierluigi Di Lizia ${ }^{\mathrm{a}}$, Mauro Massari ${ }^{\mathrm{a}}$, Alexander Wittig ${ }^{\mathrm{b}}$ \\ ${ }^{a}$ Politecnico di Milano, Via La Masa 34, 20156 Milano, Italy \\ francesco.cavenago@polimi.it·pierluigi.dilizia@polimi.it·mauro.massari@polimi.it \\ ${ }^{b}$ Astronautics Group, University of Southampton, Southampton SO17 1BJ, UK \\ a.wittig@soton.ac.uk
}

\begin{abstract}
This paper analyzes the real-time relative pose estimation and attitude prediction of a tumbling target spacecraft through a high-order numerical extended Kalman filter based on differential algebra. Indeed, in the differential algebra framework, the Taylor expansion of the phase flow is automatically available once the spacecraft dynamics is integrated and thus the need to write and integrate high-order variational equations is completely avoided making the presented solution easier to implement. To validate the technique, the ESA's e.deorbit mission, involving the Envisat satellite, is used as reference test case. The developed algorithms are implemented on a BeagleBone Black platform, as representative of the limited computational capability available on onboard processors. The performance is assessed by varying the measurement acquisition frequency and processor clock frequency, and considering various levels of uncertainties. A comparison among the different orders of the filter is carried out.

Keywords: State estimation, Kalman filter, Differential algebra, Space debris, Nonlinear filter, Pose estimation, Processor in the loop.
\end{abstract}

\section{Introduction}

The nonlinear filtering problem plays an important role in various space-related applications such as orbit determination and spacecraft navigation problems. Near future

${ }^{*}$ Corresponding author 
sample and return missions from small bodies, landing missions to the Moon, Mars and outer planets, interplanetary exploration missions, as well as relative pose estimation during rendezvous maneuvers with uncooperative targets demand navigation systems based on accurate filtering techniques that are able to perform accurate trajectory estimation in a very reduced lapse of time.

To deal with estimation problems, many filtering techniques have been developed. At present time, one of the most exploited estimation algorithm is the extended Kalman filter $^{1}(\mathrm{EKF})$. The EKF is based on the main idea of linearizing the equations of motion and the measurement equations via first-order Taylor expansions around the current mean and covariance. In some cases, however, the linear assumption may fail due to the nature of the dynamics or the frequency of available measurements, leading to inaccurate realization of the local motion. Therefore, alternative methods capable of accounting for system nonlinearity must be used. A different approach is the unscented Kalman filter ${ }^{2,3}$ (UKF). This technique is based on the unscented transformation, which does not contain any linearization, and thus provides superior performance with respect to the EKF in nonlinear problems. However, the UKF is often slightly slower than the EKF. Conversely, Park and Scheeres ${ }^{4,5}$ developed a nonlinear filter named higher-order numerical extended Kalman filter (HNEKF) by implementing a semi-analytic orbit uncertainty propagation technique, that is by solving for the higherorder Taylor series terms that describe the localized nonlinear motion and by analytically mapping the initial uncertainties. These higher-order filters are more accurate than the EKF, as the prediction step relies on a fully nonlinear mapping of the means and covariances. However, the HNEKF needs to derive the so-called higher-order tensors, which makes it in many cases - especially for a sophisticated, high fidelity system model - difficult to use due to computational complexity.

The complexity of deriving the high-order tensors and mapping the mean and covariance through the resulting polynomials can be easily solved using Differential Algebra (DA). ${ }^{6}$ DA techniques have become increasingly popular in various aerospace engineering applications over the past 5-10 years. They allow computing polynomial expansions of functions representing a dynamical system in terms of initial conditions or parameters. The calculation of these polynomials is computationally expensive, but 
can often replace many iterations of a pointwise computation or provide valuable higher order information otherwise not readily available. In particular, in ground based uncertainty propagation and sensitivity analysis, this has been shown to reduce the overall computational cost significantly when compared to alternative techniques in a wide variety of fields ranging from particle accelerator physics to astrodynamics. ${ }^{7,8,9,10,11,12}$

By substituting the classical implementation of real algebra with the implementation of a new algebra of Taylor polynomials, any function $f$ of $n$ variables can be easily expanded into its Taylor polynomial up to an arbitrarily order $m$ in the DA framework. This has a strong impact when the numerical integration of an ordinary differential equation (ODE) is performed by means of an arbitrary integration scheme. Any integration scheme is based on algebraic operations, involving the evaluation of the ODE right hand side at several integration points. Therefore, starting from the DA representation of the initial conditions and carrying out all the evaluations in the DA framework, the flow of an ODE is obtained at each step as its Taylor expansion in the initial conditions. Consequently, considering the HNEKF, by propagating the mean trajectory and evaluating the measurement function in the DA framework, we readily obtain not only their pointwise values, but also the higher-order partials. This eliminates the need to calculate the higher-order tensors at each time step by solving a complex system of augmented ODE.

Despite the evident advantages, the DA approach is not free of drawbacks. More 55 specifically, the approach relies on Taylor expansions. Thus, the problem to be dealt with must be well behaved. In addition, the computational burden associated to the calculation of the polynomials quickly increases with the order, which could limit the current applicability of the DA-based HNEKF for onboard applications. Consequently, the aim of this work is to assess the performance and onboard applicability of the DAbased HNEKF algorithm.

The target application of estimating the relative pose between two spacecraft during a rendezvous maneuver is taken into account. Camera measurements of relative position and attitude are assumed to be available. Improved estimation processes may foster an increase of autonomy in guidance, navigation and control (GNC) operations. Indeed, autonomous rendezvous and docking requires that two spacecrafts start at a 
remote distance, come together into a common orbit, rendezvous, dock, and control the new combined spacecraft in both orbit and attitude. Doing this requires developing and testing a variety of new technologies including absolute and relative autonomous navigation, autonomous rendezvous and docking hardware and software (both sensors and actuators), and autonomous control of a "new" spacecraft with different mass and inertia properties than either of the two original spacecraft. While there is substantial potential for cost savings, risk reduction, and new mission modes by use of these technologies, there is a very strong reticence to allowing operational spacecraft to control themselves. In order to defeat such reticence, much effort shall be spent to increase the reliability of the autonomous GNC strategies by improving the accuracy of the estimation algorithms and the robustness of the control laws to noise and system errors.

Focusing on the problem of rendezvous between two spacecraft, we usually think of one active spacecraft, called the chaser, and one which is a passive target, whose principal role is to remain reasonably stable while the chaser does most of the work.

so The target may be either cooperative, with some type of beacon or target and a docking fixture, if that is required, or uncooperative, i.e., a spacecraft that has no active or passive equipment, targets, or procedures to help in the process. A typical example of the latter is the case of a chaser approaching the target during an active debris removal (ADR) mission. ${ }^{13}$ Even before attempting the capture, one of the main challenges of 85 ADR missions is approaching the target, estimating the relative pose with respect to the chaser, and studying and predicting the attitude of the target, which, in the general case, tends to show a tumbling motion. This is crucial for safe proximity operation and demands accurate, real-time measurements and estimations of relative range and attitude. ${ }^{14,15}$ Generally, attitude and range estimation in rendezvous problems with cooperative and uncooperative targets can be classified into model-based and non-modelbased techniques. Non-model-based techniques match corresponding features in the two views of a stereo camera for pose estimation, not depending on a priori knowledge of the object. Many such algorithms may have difficulty in image-to-model feature correlation and foreground-background segmentation. On the contrary, model-based techniques take advantage of a priori knowledge of the object whose pose and motion are to be estimated. Thanks to the available CAD model, these techniques can compute 
the object pose and range from a single image. In both cases, due to the presence of noise and errors, the estimation method shall include filtering. Thus, the problem of relative pose estimation and attitude prediction shall go through the following steps:

1. time update of state and covariance of 6 degrees-of-freedom (DOF) motion

2. measurement update and state vector estimation (in particular the inertial attitude of the tumbling target).

In all of the above steps, the state vector must include the translational and rotational motion. More specifically, in the time update of the state and covariance, the nonlinear 6DOF dynamics, including possible couplings between the translational and rotational motion, should be considered. In addition, the state vector prediction shall aim at estimating the inertial attitude of the tumbling target. The necessity of propagating covariance information, as well as the presence of natural uncertainty in the image processing and in the mass properties of the target, highlights the need of an accurate handling of uncertainties throughout the estimation process.

Hence, the goal of this study is two-fold. First, to develop a DA-based HNEKF for relative pose estimation, considering the rendezvous with an uncooperative target, modeled after ESA's e.deorbit mission. ${ }^{16} \mathrm{~A}$ fast and accurate algorithm that provide an estimate of the uncertainty of the target state suitable for real-time operation is obtained. Afterwards, this DA algorithm is implemented on hardware mimicking the limited computational power available in space hardware. While ESA's LEON $-4^{17}$ is currently the most relevant processor for this application, our assessments is based on an ARM platform, the BeagleBone Black This platform is still representative of the limited computational capability but easier to develop for, due to wider community 120 support.

The performance and onboard applicability of the DA-based algorithm is assessed in terms of its real-time capabilities under various assumptions on the hardware. Furthermore, sensitivity analysis is performed with respect to several parameters to illustrate the conditions under which the nonlinear filters outperform traditional linear

\footnotetext{
${ }^{1}$ https://elinux.org/Beagleboard:BeagleBoneBlack
} 


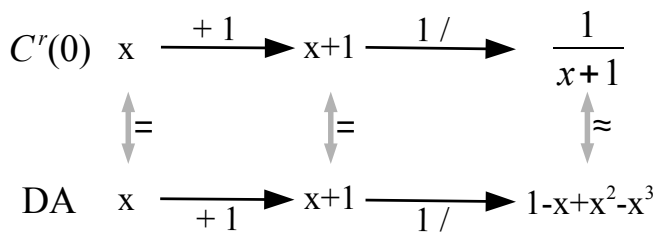

Figure 1: Evaluation of the expression $1 /(1+x)$ in $C^{r}(0)$ and DA arithmetic.

125 filtering.

The paper is organized as follows. First, an introduction to DA is given and the derivation of the DA-based HNEKF is explained. Then, a comparison among different orders of the filter is carried out. Afterwards, the considered relative pose estimation problem is introduced and the dynamics model is developed. Finally, the performance of the filters are assessed through numerical simulations.

\section{Differential algebra}

Differential Algebra techniques allow solving analytical problems through an algebraic approach. ${ }^{8}$ Similar to the computer representation of real numbers as Floating Point (FP) numbers, DA allows the representation and manipulation of functions on a computer. Each sufficiently often differentiable function $f$ is represented by its Taylor expansion around an expansion point truncated at an arbitrary finite order. Without loss of generality, we choose 0 as the expansion point. Algebraic operations on the space of truncated Taylor polynomials are defined such that they approximate the operations on the function space $C^{r}(0)$ of $r$ times differentiable functions at 0 . More specifically, each operation is defined to result in the truncated Taylor expansion of the correct result computed on the function space $C^{r}(0)$. This yields the so-called Truncated Power Series Algebra (TPSA). ${ }^{7}$

To illustrate the process, consider Fig. 1. The expression $1 /(x+1)$ is evaluated once in $C^{r}(0)$ and then in the DA framework with truncation order 3. Starting with the identity function $x$, we add one to arrive at the function $x+1$, the representation of which is fully accurate in DA as it is a polynomial of order 1. Continuing the evaluation the multiplicative inversion is performed, resulting in the function $1 /(1+x)$ in $C^{r}(0)$. 
As this function is not a polynomial any more, it is automatically approximated in DA arithmetic by its truncated Taylor expansion around 0 , given by $1-x+x^{2}-x^{3}$. Note that, by definition of the DA operations, the diagram for each single operation commutes. That is to say the same result is reached by first Taylor expanding a $C^{r}(0)$ function (moving from the top to the bottom of the diagram) and then performing the DA operation (moving from left to right), or by first performing the $C^{r}(0)$ operation and then Taylor expanding the result.

In addition to algebraic operations, the DA framework can be endowed with natural differentiation and integration operators, completing the structure of a differential algebra. Intrinsic functions, such as trigonometric and exponential functions, are built from elementary algebraic operations. ${ }^{8}$ This way, Taylor expansions of arbitrary sufficiently smooth functions given by some closed-form expression can be computed fully algebraically in a computer environment. An implementation of such DA computer routines is available in the software DACE $2.0,{ }^{18}$ which is used to implement the algorithm presented in this paper.

An important application of DA in engineering applications is the expansion of the flow $\varphi\left(t ; x_{0}\right)$ of an Ordinary Differential Equation (ODE) to arbitrary order with respect to initial conditions, integration times and system parameters. The following is a short summary of the underlying concept. For a more complete introduction to DA, as well as a fully worked out illustrative example of a DA based ODE integrator using a simple Euler step, see Valli et al. (2014). ${ }^{6}$

Consider the initial value problem

$$
\left\{\begin{array}{l}
\dot{x}=f(x, t) \\
x\left(t_{0}\right)=x_{0},
\end{array}\right.
$$

and its associated flow $\varphi\left(t ; x_{0}\right)$. By means of classical numerical integration schemes, such as Runge-Kutta or multi-step methods, it is possible to compute the orbit of a single initial condition $x_{0}$ using floating point arithmetic on a computer. Starting instead from the DA representation of an initial condition $x_{0}$, and performing all operations in the numerical integration scheme in DA arithmetic, DA allows propagating the Taylor expansion of the flow around $x_{0}$ forward in time, up to the desired final time $t_{f}$, yielding 
175 a polynomial expansion of $\varphi\left(t_{f} ; x_{0}+\delta x_{0}\right)$ up to arbitrary order.

The conversion of standard explicit integration schemes to their DA counterparts is rather straightforward. One simply replaces all operations performed during the execution of the scheme by the corresponding DA operations. Step size control and error estimates are performed only on the constant part of the polynomial, i.e. the reference trajectory of the expansion point. The result is an automatic Taylor expansion of the result of the numerical method (i.e. the numerical approximation to the flow) with respect to any quantity that was initially set to a DA value.

The main advantage of the DA-based approach is that there is no need to derive, implement and integrate variational equations in order to obtain high-order expansions of the flow. As this is achieved by merely replacing algebraic operations on floating-point numbers by DA operations, the method is inherently ODE independent. Furthermore, an efficient implementation of DA such as the DACE 2.0 package, allows us to obtain high-order expansions with limited computational time.

\section{High-order numerical extended Kalman filter}

This section is devoted to introduce the algorithm of the high-order DA-based HNEKF and to provide a first assessment of its performance. The equations of motion and measurement equations describing a generic dynamic system are as follows:

$$
\begin{aligned}
& \boldsymbol{x}_{k+1}=\boldsymbol{\Phi}\left(t_{k+1} ; \boldsymbol{x}_{k}, t_{k}\right)+\boldsymbol{w}_{k}, \\
& \boldsymbol{z}_{k+1}=\boldsymbol{h}\left(\boldsymbol{x}_{k+1}, t_{k+1}\right)+\boldsymbol{v}_{k+1},
\end{aligned}
$$

190

where $\boldsymbol{x}_{k}$ is the $m$-dimensional vector of state, $\boldsymbol{w}_{k}$ is the process noise perturbing the state, $\boldsymbol{z}_{k}$ is the $n$-dimensional vector of actual measurements, $\boldsymbol{h}$ is the measurement function, and $v_{k+1}$ is the measurement noise characterizing the observation error. The process noise and the measurement noise are assumed to be uncorrelated, that is, $E\left\{\boldsymbol{v}_{i} \boldsymbol{w}_{j}^{T}\right\}=0$, with the autocorrelations $E\left\{\boldsymbol{w}_{i} \boldsymbol{w}_{j}^{T}\right\}=\boldsymbol{Q}_{i} \delta_{i j}$ and $E\left\{\boldsymbol{v}_{i} \boldsymbol{v}_{j}^{T}\right\}=\boldsymbol{R}_{i} \delta_{i j}$ for all discrete time indexes $i$ and $j$.

Starting from the general theory of state estimation, HNEKF sequentially estimate the state and the associated uncertainty by incorporating system nonlinearity in terms 
of higher-order Taylor expansions and relying on the assumption that uncertainties can be described using Gaussian statistics.

\subsection{The DA-based HNEKF}

Consider the system model equations (2). The filtering process can be summarized as follows:

1. Prediction step: at time $t_{k+1}$, the mean and covariance of the state vector, $\boldsymbol{m}_{k+1}^{-}$ and $P_{k+1}^{-}$, and the mean of the measurements, $\boldsymbol{n}_{k+1}^{-}$, are estimated as:

$$
\begin{aligned}
m_{k+1, i}^{-} & =E\left\{\Phi_{i}\left(t_{k+1} ; \boldsymbol{x}_{k}, t_{k}\right)+w_{k, i}\right\} \\
P_{k+1, i j}^{-} & =E\left\{\left[\Phi_{i}\left(t_{k+1} ; \boldsymbol{x}_{k}, t_{k}\right)-m_{k+1, i}^{-}+w_{k, i}\right]\left[\Phi_{j}\left(t_{k+1} ; \boldsymbol{x}_{k}, t_{k}\right)-m_{k+1, j}^{-}+w_{k, j}\right]\right\} \\
n_{k+1, p}^{-} & =E\left\{h_{p}\left(\boldsymbol{x}_{k+1}, t_{k+1}\right)+v_{k+1, p}\right\}
\end{aligned}
$$

where $i, j=1, \ldots, m, p=1, \ldots, n, E\{\}$ denotes the expectation operator, and $m_{k+1, i}^{-}$, $P_{k+1, i j}^{-}$and $n_{k+1, l}^{-}$are the components of $\boldsymbol{m}_{k+1}^{-}, P_{k+1}^{-}$, and $\boldsymbol{n}_{k+1}^{-}$respectively;

2. Update step: the new measurements acquired at time $t_{k+1}, z_{k+1}$, are incorporated into the updated estimate of the state vector and covariance matrix as follows:

$$
\begin{aligned}
P_{k+1, p q}^{z z} & =E\left\{\left[h_{p}\left(\boldsymbol{x}_{k+1}, t_{k+1}\right)-n_{k+1, p}^{-}+v_{k+1, p}\right]\left[h_{p}\left(\boldsymbol{x}_{k+1}, t_{k+1}\right)-n_{k+1, q}^{-}+v_{k+1, p}\right]\right\} \\
P_{k+1, i p}^{x z} & =E\left\{\left[\Phi_{i}\left(t_{k+1} ; \boldsymbol{x}_{k}, t_{k}\right)-m_{k+1, i}^{-}+w_{k, i}\right]\left[h_{p}\left(\boldsymbol{x}_{k+1}, t_{k+1}\right)-n_{k+1, p}^{-}+v_{k+1, p}\right]\right\} \\
K_{k+1} & =P_{k+1}^{x z}\left(P_{k+1}^{z z}\right)^{-1} \\
\boldsymbol{m}_{k+1}^{+} & =\boldsymbol{m}_{k+1}^{-}+K_{k+1}\left(z_{k+1}-\boldsymbol{n}_{k+1}^{-}\right) \\
P_{k+1}^{+} & =P_{k+1}^{-}-K_{k+1} P_{k+1}^{z z} K_{k+1}^{T},
\end{aligned}
$$

where $q=1, \ldots, n, K_{k+1}$ is the Kalman gain matrix, $P_{k+1}^{x z}$ is the cross-covariance matrix of the state and the measurement, and $P_{k+1}^{z z}$ is the covariance matrix of the measurements.

In the classical EKF scheme, at each time $t_{k}$, the equations of motion and the measurement equation (2) are linearized about the current estimate of the mean and take the form:

$$
\begin{aligned}
\boldsymbol{x}_{k+1} & =\boldsymbol{m}_{k+1}^{-}+A_{k} \delta \boldsymbol{x}_{k}+\boldsymbol{w}_{k}, \\
z_{k+1} & =\boldsymbol{h}\left(\boldsymbol{m}_{k+1}^{-}, t_{k+1}\right)+C_{k} \delta \boldsymbol{x}_{k}+\boldsymbol{v}_{k+1},
\end{aligned}
$$


where $A_{k}=\frac{\partial \boldsymbol{\Phi}\left(t_{k+1} ; \boldsymbol{x}_{k}, t_{k}\right)}{\partial \boldsymbol{x}_{k}}, C_{k}=\frac{\partial \boldsymbol{h}\left(\boldsymbol{x}_{k+1}, t_{k+1}\right)}{\partial \boldsymbol{x}_{k}}$, and $\delta \boldsymbol{x}_{k}$ is the deviation of the estimated mean from the true trajectory at time $t_{k}$, i.e., $\delta \boldsymbol{x}_{k}=\boldsymbol{x}\left(t_{k}\right)-\boldsymbol{m}_{k}^{+}$, and $\boldsymbol{m}_{k+1}^{-}$is computed as

$$
\boldsymbol{m}_{k+1}^{-}=\boldsymbol{\Phi}\left(t_{k+1} ; \boldsymbol{m}_{k}^{+}, t_{k}\right)
$$

Thus, the expectation operator in Eqs. (3) and (4) can take advantage of the linearity of Eq. (5) with respect to the state.

The DA implementation of the HNEKF relies on the fact that DA can easily provide the arbitrary order Taylor expansion of both $\boldsymbol{\Phi}$ and $\boldsymbol{h}$ in Eq. (2). Thus, the arbitrary order expansion of the equations of motion and measurement equations can be easily written, and component-wise reads:

$$
\begin{aligned}
x_{k+1, i} & =\Phi_{i}\left(t_{k+1} ; \boldsymbol{m}_{k}^{+}, t_{k}\right)+\sum_{r=1}^{v} \frac{1}{r !} \Phi_{\left(t_{k+1}, t_{k}\right)}^{i, \gamma_{1} \ldots} \delta x_{k, 1}^{\gamma_{1}} \ldots \delta x_{k, m}^{\gamma_{r}}+w_{k, i}, \\
z_{k+1, p} & =h_{p}\left(\boldsymbol{\Phi}\left(t_{k+1} ; \boldsymbol{m}_{k}^{+}, t_{k}\right), t_{k+1}\right)+\sum_{r=1}^{v} \frac{1}{r !} h_{\left(t_{k+1}, t_{k}\right)}^{\left.p, \gamma_{1}\right)} \delta x_{k, 1}^{\gamma_{1}} \ldots \delta x_{k, m}^{\gamma_{r}}+v_{k+1, p},
\end{aligned}
$$

where $v$ is the order of the expansion, $\gamma_{i} \in\{1, \ldots, m\}, \Phi_{\left(t_{k+1}, t_{k}\right)}^{i, \gamma_{1} \ldots}$ includes the higher-order partials of the solution flow, which map the deviations at time $k$ to time $k+1$, and $h_{\left(t_{k+1}, t_{k}\right)}^{p, \gamma_{1} \ldots \gamma_{r}}$ includes the higher-order partials of the measurement function. Both $\Phi_{\left(t_{k+1}, t_{k}\right)}^{i, \gamma_{1} \ldots \gamma_{r}}$ and $h_{\left(t_{k+1}, t_{k}\right)}^{p, \gamma_{1}, \gamma_{r}}$ are obtained by integrating the equations of motion and evaluating the measurement equations in the DA framework.

The Taylor polynomials of Eq. (7) can be inserted into Eqs. (3) and (4) to obtain the steps of the high-order extended Kalman filter:

1. Prediction step: at time $t_{k+1}$, the mean and covariance of the state vector, $\boldsymbol{m}_{k+1}^{-}$ and $P_{k+1}^{-}$, and the mean of the measurements, $\boldsymbol{n}_{k+1}^{-}$, are estimated as:

$$
\begin{aligned}
m_{k+1, i}^{-}= & \Phi_{i}\left(t_{k+1} ; \boldsymbol{m}_{k}^{+}, t_{k}\right)+\sum_{r=1}^{v} \frac{1}{r !} \Phi_{\left(t_{k+1}, t_{k}\right)}^{i, \gamma_{1} \ldots \gamma_{r}} E\left\{\delta x_{k, 1}^{\gamma_{1}} \ldots \delta x_{k, m}^{\gamma_{r}}\right\} \\
P_{k+1, i j}^{-}= & \sum_{r=1}^{v} \sum_{s=1}^{v} \frac{1}{r ! s !} \Phi_{\left(t_{k+1}, t_{k}\right)}^{i, \gamma_{1} \ldots \gamma_{r}} \Phi_{\left(t_{k+1}, t_{k}\right)}^{j, \xi_{1} \ldots \xi_{s}} E\left\{\delta x_{k, 1}^{\gamma_{1}} \ldots \delta x_{k, m}^{\gamma_{r}} \delta x_{k, 1}^{\xi_{1}} \ldots \delta x_{k, m}^{\xi_{s}}\right\}+ \\
& -\delta m_{k+1}^{i} \delta m_{k+1}^{j}+Q_{k}^{i j} \\
n_{k+1, p}^{-}= & h_{p}\left(\boldsymbol{\Phi}\left(t_{k+1} ; \boldsymbol{m}_{k}^{+}, t_{k}\right), t_{k+1}\right)+\sum_{r=1}^{v} \frac{1}{r !} h_{\left(t_{k+1}, \gamma_{k}\right)}^{p, \gamma_{1} \ldots \gamma_{r}} E\left\{\delta x_{k, 1}^{\gamma_{1}} \ldots \delta x_{k, m}^{\gamma_{r}}\right\},
\end{aligned}
$$

where $\xi_{i} \in\{1, \ldots, m\}$ and $\delta m_{k+1}^{i}=\Phi_{i}\left(t_{k+1} ; \boldsymbol{m}_{k}^{+}, t_{k}\right)-m_{k+1, i}^{-}$;

2. Update step: the new measurements acquired at time $t_{k+1}, z_{k+1}$, are incorporated 
into the updated estimate of the state vector and covariance matrix as follows:

$$
\begin{aligned}
P_{k+1, p q}^{z z}= & \sum_{r=1}^{v} \sum_{s=1}^{v} \frac{1}{r ! s !} h_{\left(t_{k+1}, t_{k}\right)}^{p, \gamma_{1} \ldots \gamma_{r}} h_{\left(t_{k+1}, t_{k}\right)}^{q, \xi_{1} \ldots \xi_{s}} E\left\{\delta x_{k, 1}^{\gamma_{1}} \ldots \delta x_{k, m}^{\gamma_{r}} \delta x_{k, 1}^{\xi_{1}} \ldots \delta x_{k, m}^{\xi_{s}}\right\}+ \\
& -\delta n_{k+1}^{p} \delta n_{k+1}^{q}+R_{k+1}^{p q} \\
P_{k+1, i p}^{x z}= & \sum_{r=1}^{v} \sum_{s=1}^{v} \frac{1}{r ! s !} \Phi_{\left(t_{k+1}, t_{k}\right)}^{i, \gamma_{1} \ldots \gamma_{r}} h_{\left(t_{k+1}, t_{k}\right)}^{p, \xi_{1} \ldots \xi_{s}} E\left\{\delta x_{k, 1}^{\gamma_{1}} \ldots \delta x_{k}^{\gamma_{r}} \delta x_{k, m}^{\xi_{1}} \ldots \delta x_{k}^{\xi_{s}}\right\}+ \\
& -\delta m_{k+1}^{i} \delta n_{k+1}^{p} \\
K_{k+1}= & P_{k+1}^{x z}\left(P_{k+1}^{z z}\right)^{-1} \\
\boldsymbol{m}_{k+1}^{+}= & \boldsymbol{m}_{k+1}^{-}+K_{k+1}\left(\boldsymbol{z}_{k+1}-\boldsymbol{n}_{k+1}^{-}\right) \\
P_{k+1}^{+}= & P_{k+1}^{-}-K_{k+1} P_{k+1}^{z z} K_{k+1}^{T},
\end{aligned}
$$$$
\text { where } \delta n_{k+1}^{p}=h_{p}\left(\boldsymbol{\Phi}\left(t_{k+1} ; \boldsymbol{m}_{k}^{+}, t_{k}\right), t_{k+1}\right)-n_{k+1, p}^{-} .
$$

If the case of variables with Gaussian random distributions is considered, the higherorder moments $E\left\{\delta x_{k}^{\gamma_{1}} \ldots \delta x_{k}^{\gamma_{p}}\right\}$ can be completely described by the first two moments (i.e., mean and covariance), and can be easily computed in terms of the covariance matrix using Isserlis' formula on the monomials of the Taylor polynomial. ${ }^{19}$ It is worth stressing that, in order to apply standard HNEFK, each high-order partial in Eq. (8) is computed by integrating for each time interval a dedicated differential equation that must be derived analytically (see Park and Scheeres ${ }^{4}$ ).Conversely, in the DA framework, this operation is completely avoided since the high-order partials are automatically obtained from the DA-based integration of the dynamic system ${ }^{6}$ (Eq. (2)).

\subsection{Order comparison}

Before applying the DA-based HNEKF to the relative pose estimation problem, the effects of considering high-order expansion of the dynamic flow in the extended Kalman filter is discussed.

Consider the illustrative example of an Earth-orbiting spacecraft. The second-order differential equation governing the motion is

$$
\frac{d \dot{\boldsymbol{r}}}{d t}=-\frac{\mu}{r^{3}} \boldsymbol{r},
$$

where $\boldsymbol{r}$ is the position vector of the spacecraft and $\mu$ is the Earth gravitational parameter. It is assumed that there is no external disturbing force for the system except the 
gravitational force between the Earth and the spacecraft. The initial true position and velocity assumed for this test are

$$
\boldsymbol{x}_{0}=\left(\begin{array}{l}
\boldsymbol{r}_{0} \\
\boldsymbol{v}_{0}
\end{array}\right)=\left(\begin{array}{c}
-0.68787 \\
-0.39713 \\
+0.28448 \\
-0.51331 \\
+0.98266 \\
+0.37611
\end{array}\right),
$$

where the length units are scaled by the orbit semi-major axis $(a=8788 \mathrm{~km})$ and the time by $\sqrt{\frac{a^{3}}{\mu}}$. The initial estimates for the state are $10 \%$ off from the true initial state values shown in Eq. 11. The adopted initial error covariance is a diagonal matrix with variance 0.01 for the position vector components and $10^{-4}$ for the velocity vector components. The measurements used in the simulation are the radial position of the spacecraft with respect to the Earth and the line of sight directions to the planet:

$$
\begin{aligned}
& z_{1}=r+v_{1} \\
& z_{2}=\arctan \left(\frac{y}{x}\right)+v_{2} \\
& z_{3}=\arcsin \left(\frac{z}{r}\right)+v_{3},
\end{aligned}
$$

where $v_{i}$, represents the measurement noise. The standard deviation of the measurement error for the radial position is assumed to be $10^{-3} \mathrm{~km}$. The angle error is assumed to be $1.74510^{-6}$ rad following the conventional measurement accuracy for the directional measurement. No process noise is included.

The position and velocity error profiles obtained with the DA-based HNEKF using first, second, and third order expansions are compared in Figs. 2 and 3 The position and velocity errors, $\epsilon_{r}$ and $\epsilon_{v}$ respectively, are defined as the Euclidean norm of the difference vector between the estimated position and velocity, and the corresponding true vectors. In one orbit a total number of 12 measurements separated by equal time intervals is considered. Note that the results obtained with first order expansions are representative of the performance of a classical EKF scheme. The simulation results show that the estimation accuracy significantly improves when we move from a first order to a second order filter. This confirms that the higher order filters can extract 


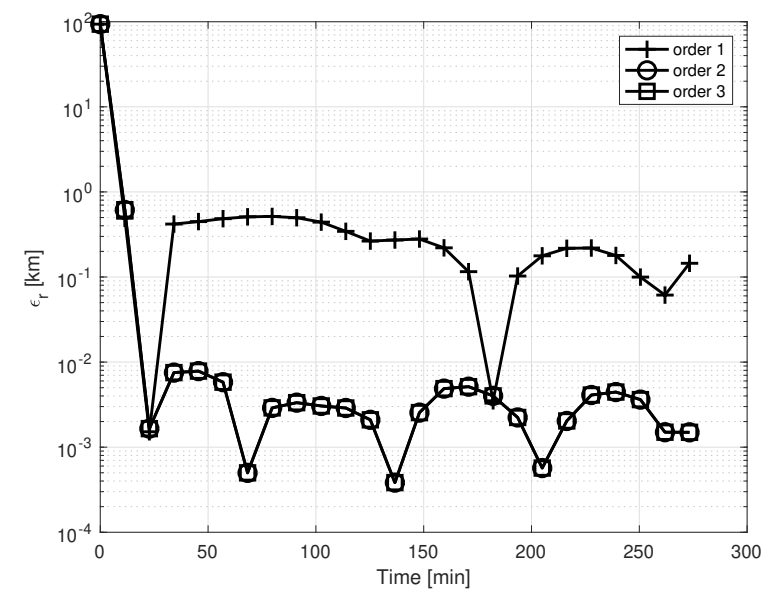

Figure 2: Orbit determination on Keplerian dynamics, 12 measurements per orbit: position error profiles of the DA-based HNEKF at different orders.

more information from the available nonlinear measurements compared to the first order filter. On the other hand, Figs. 2 and 3 show that there is no accuracy gain when we use the third order filter, as the errors profiles corresponding to the second and third order basically overlaps.

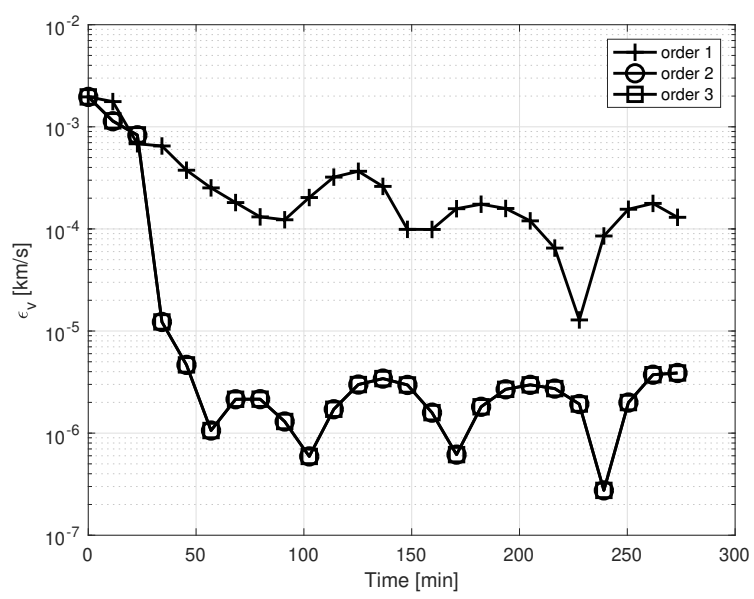

Figure 3: Orbit determination on Keplerian dynamics, 12 measurements per orbit: velocity error profiles of the DA-based HNEKF at different orders. 
Figures 4 and 5 show the standard deviation profiles for the spacecraft position and velocity. More specifically, the figures report the quantities

$$
\begin{aligned}
\sigma_{r} & =\sqrt{\sigma_{x x}^{2}+\sigma_{y y}^{2}+\sigma_{z z}^{2}} \\
\sigma_{v} & =\sqrt{\sigma_{v x}^{2}+\sigma_{v y}^{2}+\sigma_{v z}^{2}},
\end{aligned}
$$

where $\sigma_{x x}^{2}, \sigma_{y y}^{2}, \sigma_{z z}^{2}, \sigma_{v x}^{2}, \sigma_{v y}^{2}, \sigma_{v z}^{2}$ are the diagonal terms of the estimated covariance matrix.

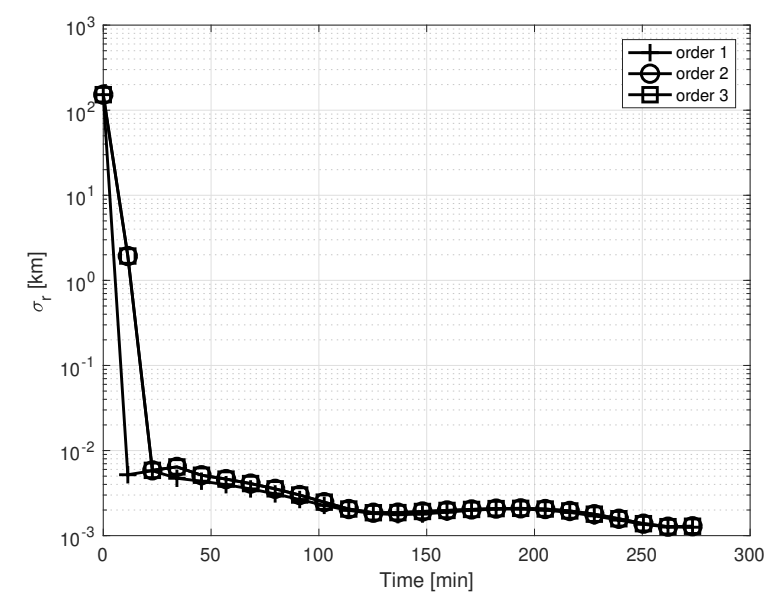

Figure 4: Orbit determination on Keplerian dynamics, 12 measurements per orbit: $\sigma_{r}$ profiles of the DAbased HNEKF at different orders.

The filter shows the same behaviour in terms of accuracy gain when different observation frequencies are adopted. Figures 6 and 7 show the position error profiles obtained when a total number of 6 and 24 measurements separated by equal time intervals is considered. As can be seen, the results confirm that the estimation accuracy significantly improves when we move from a first to a second order filter and that no further gain is obtained with the third order filter.

The lack of a significant improvement in terms of accuracy between the second and third order filters lies in the basic assumption of Kalman filters, i.e. in the hypothesis that all random distributions are Gaussian and, then, completely described by their mean and covariance. 


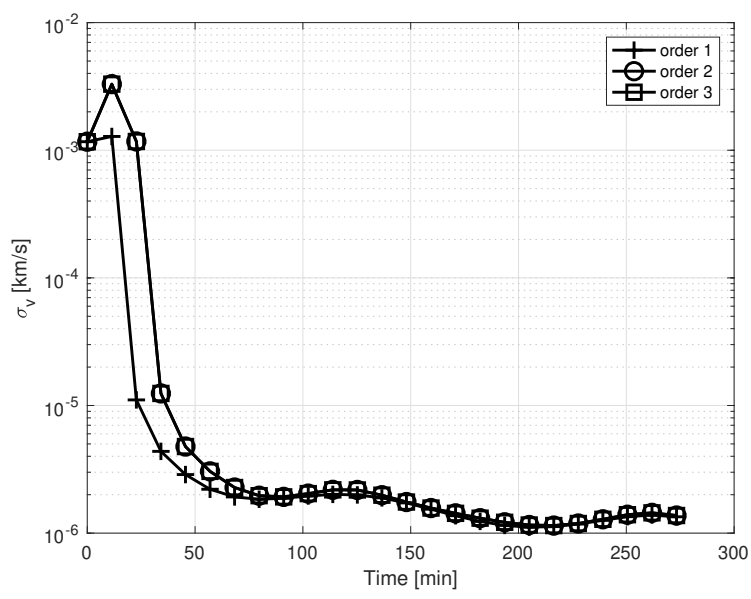

Figure 5: Orbit determination on Keplerian dynamics, 12 measurements per orbit: $\sigma_{v}$ profiles of the DAbased HNEKF at different orders.

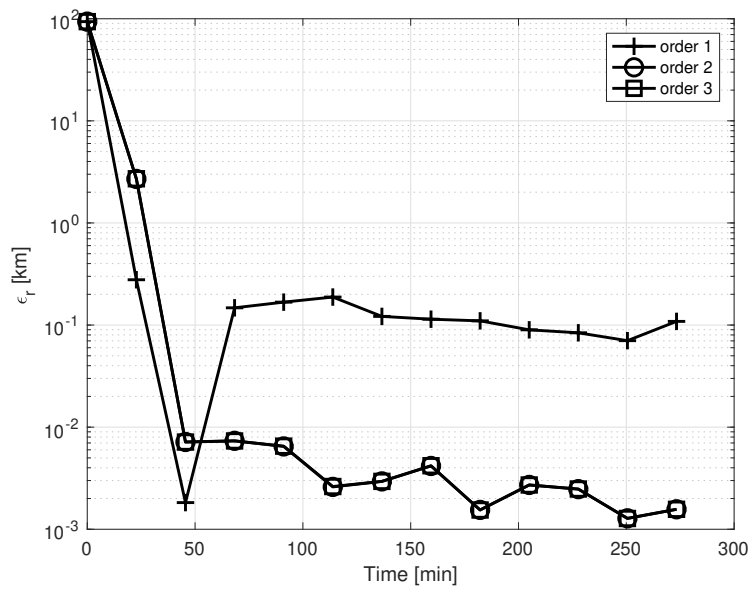

Figure 6: Orbit determination on Keplerian dynamics, 6 measurements per orbit: position error profiles of the DA-based HNEKF at different orders. 


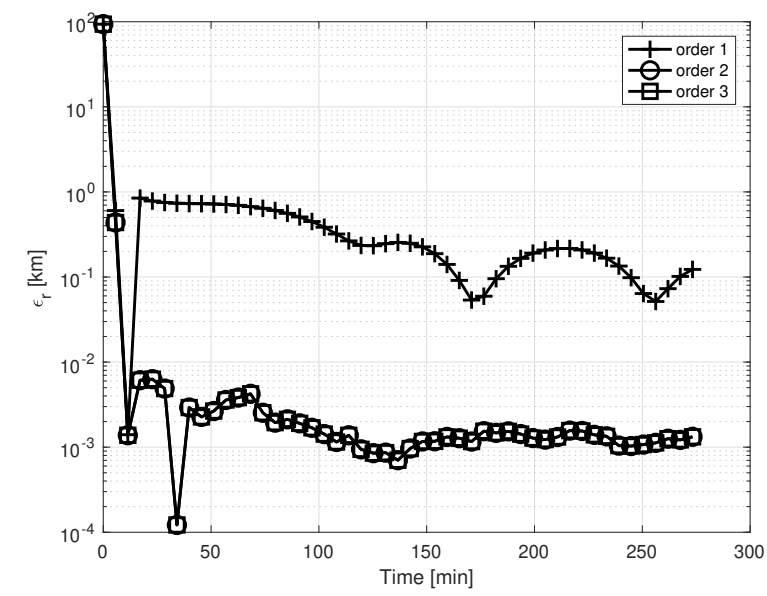

Figure 7: Orbit determination on Keplerian dynamics, 24 measurements per orbit: position error profiles of the DA-based HNEKF at different orders.

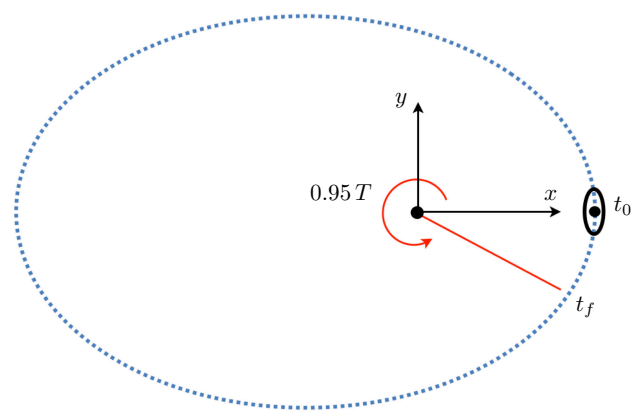

Figure 8: Schematic representation of the illustrative example on the Keplerian dynamics.

To get a deeper insight, consider a spacecraft at the pericenter of an elliptical orbit of eccentricity $e=0.5$, moving in Keplerian dynamics (see Fig. 8 for a schematic representation of the example). Assume the lengths are scaled by the orbit pericenter 


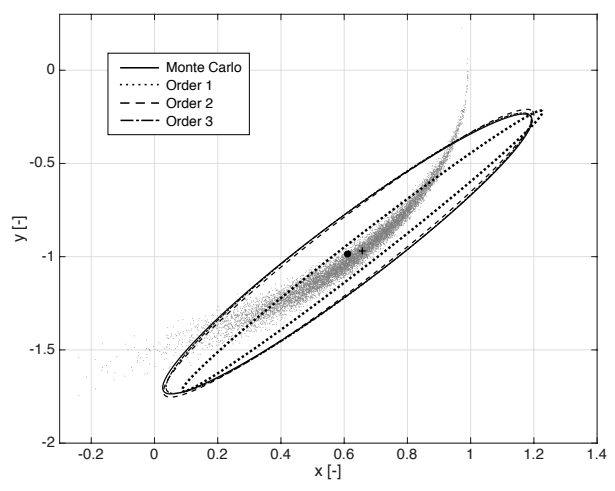

Figure 9: Propagated mean and covariance for the illustrative example on Keplerian dynamics: comparison between a Monte Carlo simulation and the DA-based estimation at different orders. Grey dots represent the propagated samples of the Monte Carlo simulation.

$r_{p}$ and the time by $\sqrt{r_{p}^{3} / \mu}$. Thus, the nominal initial state is:

$$
\begin{aligned}
& x_{0}=1 \\
& y_{0}=0 \\
& z_{0}=0 \\
& v_{x_{0}}=0 \\
& v_{y_{0}}=\sqrt{1+e} \\
& v_{z_{0}}=0,
\end{aligned}
$$

The initial position of the spacecraft is assumed to be uncertain with standard deviations $3 \sigma_{x}=0.008$ and $3 \sigma_{y}=0.08$ on the $x$ and $y$ components of the position vector, with no correlation between the different components. The uncertain initial state is propagated forward to the final epoch $t_{f}=0.95 T$, where $T=2 \pi$ is the nominal period of the orbit. First of all, a Monte Carlo simulation is carried out to propagate $10^{5}$ initial conditions to $t_{f}$ and to compute the resulting mean and covariance, which are used as reference for the following analysis. As can be seen from Fig. 9, the samples of the Monte Carlo simulation at $t_{f}$ exhibit an evident nonlinear distribution.

Using the techniques introduced in Sect. 2. DA is then used to compute arbitrary order Taylor expansions of the spacecraft state at $t_{f}$ with respect to $x_{0}$ and $y_{0}$. The 
resulting polynomials are used to compute the propagated mean and covariance using the formulas of Eq. (8). Figure 9 reports the results obtained for different expansion orders. As shown in the figure, the first order expansion fails to accurately estimate the exact mean and covariance, which are represented by the result of the Monte Carlo simulation. The second order expansion already introduces sufficient information for an accurate representation of both moments. The third order expansion provides only a slight further improvement in terms of accuracy. Thus, being based on a Gaussian representation of the propagated uncertainties, the accuracy of the extended Kalman filter significantly benefits of a second order expansion of the flow of the dynamics. However, no relevant improvement is obtained with higher orders.

The importance of higher order partials emerges in the computation of higher order moments. The DA-based estimates of the mean, covariance, skewness, and kurtosis are compared with their values obtained with the Monte Carlo simulation in Table 1 . More specifically, the following moments are computed: ${ }^{20}$

285

1. Mean: $\mu=E\left\{x\left(t_{f}\right)\right\}$

2. Variance: $\sigma^{2}=E\left\{\left[x\left(t_{f}\right)-\mu\right]^{2}\right\}$

3. Skewness: $\gamma=E\left\{\left[x\left(t_{f}\right)-\mu\right]^{3}\right\} / \sigma^{3}$

4. Kurtosis: $\kappa=E\left\{\left[x\left(t_{f}\right)-\mu\right]^{4}\right\} / \sigma^{4}-3$.

Note that, in the DA-based computation of the moments, $x\left(t_{f}\right)$ is replaced by the corresponding Taylor expansion. Thus, similarly to Eq. (8), the computation of the above expectations reduces to the computation of the expectation of the resulting monomials, which can be addressed using Isserlis' formula. ${ }^{21}$ As can be seen from Table 1 . the second order expansion is sufficient to obtain an accurate estimate of the variance. However, a third order expansion is needed to adequately approximate the skewness and the kurtosis.

Based on these results, the assessment of the performance of the DA-based HNEKF will be limited to the use of first and second order expansions in the following analyses. 
Table 1: DA-based estimates of the moments for the illustrative example on Keplerian dynamics.

\begin{tabular}{ccccc}
\hline \hline Moment & Monte Carlo & Order 1 & Order 2 & Order 3 \\
\hline$\mu$ & 0.6143 & 0.6574 & 0.6142 & 0.6142 \\
$\sigma$ & 0.0366 & 0.0353 & 0.0373 & 0.0363 \\
$\gamma$ & -0.5638 & 0 & -0.5548 & -0.5662 \\
$\kappa$ & 0.2545 & 0 & 0.4247 & 0.2214 \\
\hline
\end{tabular}

\section{Relative Pose Estimation}

This paper focuses on exploiting the proposed DA-based HNEKF to face the challeng-

$$
\boldsymbol{r}_{r}=x \hat{\boldsymbol{r}}+y \hat{\boldsymbol{\theta}}+z \hat{\boldsymbol{h}}
$$




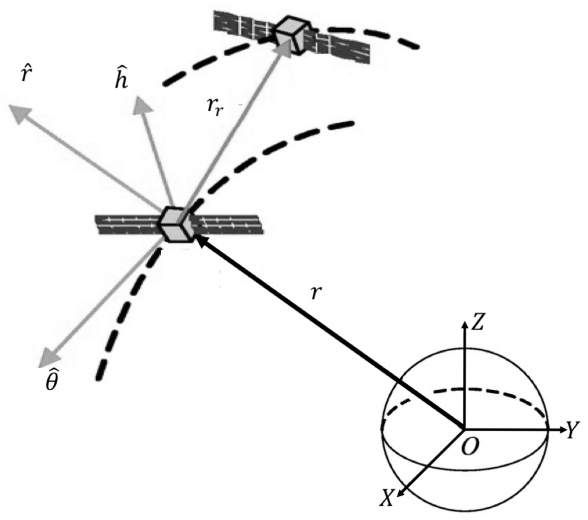

Figure 10: Chaser local vertical local horizontal frame.

$$
\boldsymbol{v}_{r}=\dot{x} \hat{\boldsymbol{r}}+\dot{y} \hat{\boldsymbol{\theta}}+\dot{z} \hat{\boldsymbol{h}}
$$

where $x, y$ and $z$ are the three components of $\boldsymbol{r}_{r}$ in the chaser LVLH frame and $\hat{\boldsymbol{r}}, \hat{\boldsymbol{\theta}}$ and $\hat{\boldsymbol{h}}$ are the versors of the considered triad. The relative translational dynamics are governed by the following equations: ${ }^{22}$

$$
\begin{gathered}
\ddot{x}-2 \dot{v} \dot{y}-\ddot{v} y-\dot{v}^{2} x=-\mu(\bar{r}+x) /\left[(\bar{r}+x)^{2}+y^{2}+z^{2}\right]^{3 / 2}+\mu / \bar{r}^{2} \\
\ddot{y}+2 \dot{v} \dot{x}+\ddot{v} x-\dot{v}^{2} y=-\mu y /\left[(\bar{r}+x)^{2}+y^{2}+z^{2}\right]^{3 / 2} \\
\ddot{z}=-\mu z /\left[(\bar{r}+x)^{2}+y^{2}+z^{2}\right]^{3 / 2}
\end{gathered}
$$

where $\mu$ is the gravitational parameter, $\bar{r}$ is the distance from the Earth center to the chaser and $v$ is the true anomaly. Finally, the motion of the chaser is described by the following equations:

$$
\begin{gathered}
\ddot{\vec{r}}=\bar{r} \dot{v}^{2}-\mu / \bar{r}^{2} \\
\ddot{v}=-2 \dot{\bar{r}} \dot{v} / \bar{r}
\end{gathered}
$$




\subsection{Relative rotational dynamics}

As for the rotational dynamics, the relative orientation of the body-fixed reference

325 frame on the target with respect to the body-fixed reference frame on the chaser can be described through a rotation matrix $\boldsymbol{\Gamma}$. Consequently, the relative angular velocity and acceleration of the target can be expressed as follows: ${ }^{23}$

$$
\begin{gathered}
\omega_{r}=\omega_{t}-\Gamma \omega_{c} \\
\dot{\omega}_{r}=\dot{\omega}_{t}-\Gamma \dot{\omega}_{c}+\dot{\omega}_{a p p} \\
\dot{\omega}_{a p p}=\omega_{r} \times \Gamma \omega_{c}
\end{gathered}
$$

where $\omega_{c}$ and $\omega_{t}$ are the angular velocity of the chaser and the target expressed in their body-fixed reference frame, respectively, whereas $\omega_{r}$ is the relative angular velocity expressed in the target body-fixed reference frame.

The relative attitude of the target can be described parameterizing the rotation matrix $\boldsymbol{\Gamma}$. To this aim, the Modified Rodrigues Parameters (MRP) are adopted in this study. ${ }^{24}$ The MRP are related to quaternions and to the rotation matrix by the following relations:

$$
\begin{gathered}
\zeta=\frac{\tilde{\boldsymbol{q}}}{1+\boldsymbol{q}_{0}} \\
\boldsymbol{\Gamma}(\boldsymbol{\zeta})=\boldsymbol{I}_{3}-\alpha_{1}^{A}[\zeta \times]+\alpha_{2}^{A}[\zeta \times]^{2} \\
\left\{\begin{array}{l}
\alpha_{1}^{A}=4 \frac{1-\zeta^{T} \zeta}{\left(1+\zeta^{T} \zeta\right)^{2}} \\
\alpha_{2}^{A}=8 \frac{1}{\left(1+\zeta^{T} \zeta\right)^{2}}
\end{array}\right.
\end{gathered}
$$

where $\zeta$ are the MRP, $\tilde{\boldsymbol{q}}$ are the quaternions and $\boldsymbol{I}_{3}$ is the identity matrix.

The time evolution of the MRP is governed by Eq. 28

$$
\dot{\zeta}=\frac{1}{4} \Sigma(\zeta) \omega_{r}
$$




$$
\Sigma(\zeta)=\left(1-\zeta^{T} \zeta\right) \boldsymbol{I}_{3}+2 \zeta \zeta^{T}+2[\zeta \times]
$$

As for the dynamics, the chaser motion is described by the torque-free Euler equations, while the relative attitude dynamics can be obtained substituting kinematics relationship in the Euler absolute equations of the target spacecraft. The resulting dynamic system is:

$$
\begin{gathered}
\boldsymbol{J}_{t} \dot{\boldsymbol{\omega}}_{r}+\boldsymbol{\omega}_{r} \times \boldsymbol{J}_{t} \boldsymbol{\omega}_{r}=\boldsymbol{M}_{\text {app }}-\boldsymbol{M}_{g}-\boldsymbol{M}_{c i} \\
\boldsymbol{M}_{\text {app }}=\boldsymbol{J}_{t} \boldsymbol{\omega}_{r} \times \boldsymbol{\Gamma} \boldsymbol{\omega}_{c} \\
\boldsymbol{M}_{c i}=\boldsymbol{J}_{t} \boldsymbol{\Gamma} \dot{\boldsymbol{\omega}}_{c} \\
\boldsymbol{M}_{g}=\boldsymbol{M}_{g c}+\boldsymbol{M}_{g \text { coup }} \\
\boldsymbol{M}_{g c}=\boldsymbol{\Gamma} \boldsymbol{\omega}_{c} \times \boldsymbol{J}_{t} \boldsymbol{\Gamma} \boldsymbol{\omega}_{c} \\
\boldsymbol{M}_{\text {gcoup }}=\left(\boldsymbol{\omega}_{r} \times \boldsymbol{J}_{t} \boldsymbol{\Gamma} \boldsymbol{\omega}_{c}+\boldsymbol{\Gamma} \boldsymbol{\omega}_{c} \times \boldsymbol{J}_{t} \boldsymbol{\omega}_{r}\right)
\end{gathered}
$$

where $\boldsymbol{J}_{t}$ is the matrix of inertia of the target, $\boldsymbol{M}_{a p p}$ is the apparent torques, $\boldsymbol{M}_{c i}$ is the chaser-inertial torques and $\boldsymbol{M}_{g}$ is the gyroscopic torques.

\subsection{Measurement model}

In real applications, relative position and relative attitude measurements can be ob-

tained by processing images from a camera. In this study, they are generated numerically exploiting a suitable error model.

While the relative position is already part of the state vector, and thus it is linearly related to it, the attitude is provided in terms of roll, pitch and yaw angles. Consequently, it is necessary to derive the rotation matrix $\boldsymbol{\Gamma}$ from the MRP (see Eq. 26]) and 

tion:

$$
\begin{gathered}
\phi=\arctan (\boldsymbol{\Gamma}(3,2) / \boldsymbol{\Gamma}(3,3)) \\
\theta=\arctan (\boldsymbol{\Gamma}(2,1) / \boldsymbol{\Gamma}(1,1)) \\
\psi=\arcsin (-\boldsymbol{\Gamma}(3,1))
\end{gathered}
$$

where $\phi, \theta$ and $\psi$ are the roll, pitch and yaw angles, respectively, whereas $\boldsymbol{\Gamma}(i, j)$ is the component of the rotation matrix $\boldsymbol{\Gamma}$ in the position $(i, j)$. It can be easily observed that the relations binding the MRP and the measured attitude introduce other nonlinearities 355 in the problem.

For the measurements generation, the true states of the target spacecraft are computed during the integration of the dynamic equations (see Secs. 4.14 4.2). Then, the measured quantities are derived as previously explained and noise is introduced by adopting the exponentially correlated random variable model ${ }^{25}$

$$
\begin{gathered}
E\left(t_{k+1}\right)=K E\left(t_{k}\right)+\sqrt{1-K^{2}} \cdot \mathcal{N}(0, \sigma) \\
K=e^{-1 /\left(f_{a} \tau\right)}
\end{gathered}
$$

360 where $E$ is the error w.r.t. the true states, $\mathcal{N}(0, \sigma)$ is a random number generated with a normal distribution of zero mean and standard deviation $\sigma, f_{a}$ is the measurement acquisition frequency and $\tau$ is the autocorrelation time. In this model the error at time $k+1$ is exponentially correlated to the error at the previous instant and this correlation decays with a time scale defined by $\tau$. Considering a camera, this seems to be a more reasonable model with respect to the Gaussian one in which error values at different time instant are completely uncorrelated. 


\subsection{Software architecture}

Fig. 11 reports the software architecture, which is made up of three main blocks. The first one is the "dynamics simulator+noise generator" that receives as inputs the initial states, then propagates the dynamics through a variable-step integrator (RungeKutta78) and generates the measurements adding noise computed with the exponentially correlated random model. These computations are performed in advance and the outputs are loaded in memory before running the filter.

For the filtering, the decoupling of the dynamics is exploited to split the problem into two parts: the estimation of the relative translational states $\left(\boldsymbol{r}_{r}\right.$ and $\left.\boldsymbol{v}_{r}\right)$ and the estimation of the relative rotational states $\left(\zeta\right.$ and $\left.\omega_{r}\right)$. In this way, six DA variables have to be initialized for each filter instead of twelve, lightening the computational burden. In both filters the required measurements and chaser absolute state are loaded at the beginning and an initial estimate of the relative states, in terms of mean and covariance, has to be provided. For the relative dynamics propagation inside the filter, a $4^{\text {th }}$-order Runge-Kutta integrator is exploited since it is a better solution for embedded systems in terms of computational effort.

Finally, the estimated relative state is compared with the true state propagated by the dynamics simulator to assess the performance of the filters.

\section{Results}

In the numerical analysis, the chaser and the target are assumed to lie on the same orbit at a distance that is compatible with the camera performance. The mass properties of the two spacecraft are listed in Table 2. The initial conditions of the relative states are reported in Table 3. The attitude is initialized randomly, while the angular velocity is selected in order to have an absolute value of about $2.5 \mathrm{deg} / \mathrm{s}$.

In the following sections, first, the accuracy and robustness of the first and second order filter are assessed, and then an analysis on the required computational time is performed in order to verify the real-time feasibility. 


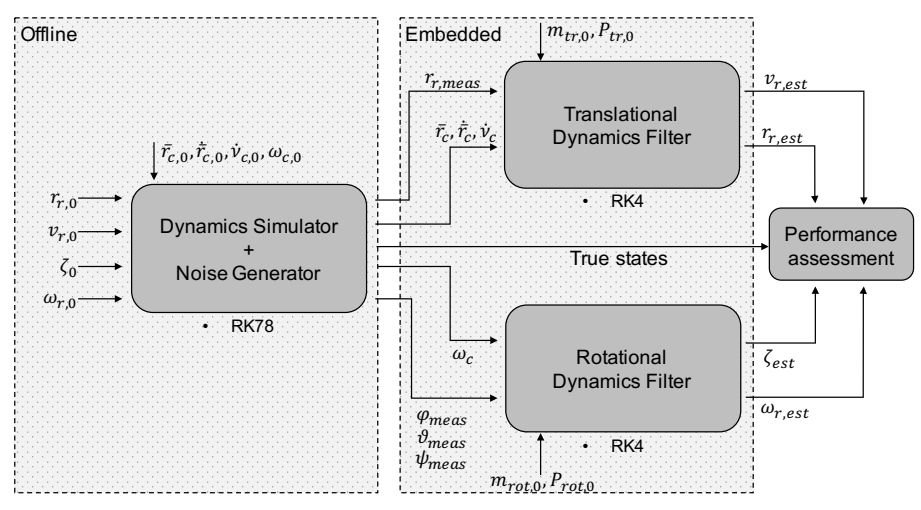

Figure 11: Software architecture.

Table 2: Mass properties.

\begin{tabular}{lcc}
\hline & Chaser & Target \\
\hline$M(\mathrm{~kg})$ & 1435 & 7828.867 \\
$J_{x x}\left(\mathrm{kgm}^{2}\right)$ & 2040 & 17023.3 \\
$J_{y y}\left(\mathrm{kgm}^{2}\right)$ & 1670 & 124825.7 \\
$J_{z z}\left(\mathrm{kgm}^{2}\right)$ & 2570 & 129112.2 \\
$J_{x y}\left(\mathrm{kgm}^{2}\right)$ & 130 & 397.1 \\
$J_{x z}\left(\mathrm{kgm}^{2}\right)$ & 25 & -2171.4 \\
$J_{y z}\left(\mathrm{kgm}^{2}\right)$ & -55 & 344.2 \\
\hline
\end{tabular}


Table 3: Initial conditions.

\begin{tabular}{llll}
\hline Tr. Dyn. & \multicolumn{3}{l}{ Rot. Dyn. } \\
\hline$x(\mathrm{~m})$ & -0.002 & $\phi(\mathrm{rad})$ & 1.66 \\
$y(\mathrm{~m})$ & -31.17 & $\theta(\mathrm{rad})$ & 2.27 \\
$z(\mathrm{~m})$ & 0 & $\psi(\mathrm{rad})$ & -0.38 \\
$\dot{x}(\mathrm{~m} / \mathrm{s})$ & $-3.5 \mathrm{e}-6$ & $\omega_{r, x}(\mathrm{rad} / \mathrm{s})$ & 0.02 \\
$\dot{y}(\mathrm{~m} / \mathrm{s})$ & $-2.0 \mathrm{e}-6$ & $\omega_{r, y}(\mathrm{rad} / \mathrm{s})$ & 0.02 \\
$\dot{z}(\mathrm{~m} / \mathrm{s})$ & 0 & $\omega_{r, z}(\mathrm{rad} / \mathrm{s})$ & 0.04 \\
\hline
\end{tabular}

\subsection{Accuracy and robustness analysis}

395 the following analyses. First, the target velocity can be assumed to be the most uncertain variables since neither a priori knowledge nor direct measurements are available. Then, low measurement acquisition frequencies could be required (or are at least beneficial) for limited-resource systems. Therefore, a Monte Carlo-based sensitivity analysis is carried out to assess the robustness of the first and second order filter with various acquisition frequency and initial uncertainty in the relative velocity. In addition, the effects of different initial linear and angular position uncertainties, initial angular rate and level of measurement noise are investigated. The examined cases are reported in Table 4 for the translational filter and in Tables 5 for the rotational filter, being $\sigma_{i, 0}$ and $\sigma_{i}^{s}$ the initial standard deviation and the sensor standard deviation, respectively, of the variable $i$.

Particular attention is paid on the performance of the rotational filter since it has to deal with more complex dynamics. Indeed, the translational dynamics are very slow and almost linear and, thus, less significant results are expected, as it will be shown 410 further.

For each case, 1000 samples are generated around the true initial conditions, according to the statistics, and then the furthest 100 are selected and used as initial estimates of the relative states in the filter. This choice is motivated by the will to study the 
Table 4: Translational dynamics: sensitivity to initial velocity uncertainty and acquisition frequency.

\begin{tabular}{lllll}
\hline \hline Dynamics & & Sensors & \multicolumn{2}{c}{ Frequency } \\
\hline$\sigma_{r_{r}, 0}(\mathrm{~m})$ & 1 & $\sigma_{x, y}^{s}(\mathrm{~m})$ & 0.02 & $0.1 \mathrm{~Hz}$ to $3 \mathrm{~Hz}$ \\
$\sigma_{v_{r}, 0}(\mathrm{~m} / \mathrm{s})$ & $\mathrm{K}^{*} 0.1$ & $\sigma_{z}^{s}(\mathrm{~m})$ & 0.03 & \\
\hline $\mathrm{K}=[0.1,0.5,1,5,10]$ & & &
\end{tabular}

Table 5: Rotational dynamics: sensitivity to angular velocity uncertainty and acquisition frequency

\begin{tabular}{|c|c|c|c|c|c|c|}
\hline \multicolumn{2}{|l|}{ Dynamics } & \multicolumn{2}{|l|}{ Sensors } & \multicolumn{2}{|c|}{ Initial Conditions } & \multirow{2}{*}{$\begin{array}{l}\text { Frequency } \\
0.1 \mathrm{~Hz}\end{array}$} \\
\hline$\sigma_{\zeta, 0}()$ & $0.002-0.02$ & $\sigma_{\phi, \theta}^{s}(\mathrm{rad})$ & 0.003 & $\omega_{r, x}(\mathrm{rad} / \mathrm{s})$ & 0.1 & \\
\hline$\sigma_{\omega_{r}, 0}(\mathrm{rad} / \mathrm{s})$ & $\mathrm{K} * 0.01$ & $\sigma_{\psi}^{s}(\mathrm{rad})$ & 0.006 & $\omega_{r, y}(\mathrm{rad} / \mathrm{s})$ & 0.2 & to $3 \mathrm{~Hz}$ \\
\hline & & & & $\omega_{r, z}(\mathrm{rad} / \mathrm{s})$ & 0.2 & \\
\hline
\end{tabular}

$\mathrm{K}=[0.1,0.5,1,5,10]$

Table 6: Rotational dynamics: sensitivity to level of measurement noise.

\begin{tabular}{llllll}
\hline \hline & Dynamics & & Sensors & & Frequency \\
\hline case 1 & $\sigma_{\zeta, 0}()$ & $\mathrm{G}^{*} 0.002$ & $\sigma_{\phi, \theta}^{s}(\mathrm{rad})$ & $\mathrm{G}^{*} 0.003$ & $3 \mathrm{~Hz}$ \\
& $\sigma_{\omega_{r}, 0}(\mathrm{rad} / \mathrm{s})$ & 0.01 & $\sigma_{\psi}^{s}(\mathrm{rad})$ & $\mathrm{G}^{*} 0.006$ & \\
case 2 & $\sigma_{\zeta, 0}()$ & $\mathrm{G}^{*} 0.002$ & $\sigma_{\phi, \theta}^{s}(\mathrm{rad})$ & $\mathrm{G}^{*} 0.003$ & $0.4 \mathrm{~Hz}$ \\
& $\sigma_{\omega_{r}, 0}(\mathrm{rad} / \mathrm{s})$ & 0.01 & $\sigma_{\psi}^{s}(\mathrm{rad})$ & $\mathrm{G}^{*} 0.006$ & \\
\hline \multicolumn{5}{c}{} & $G=[1,2,4]$ \\
\end{tabular}


worst circumstances, in which the nonlinearities are expected to play a prominent role.

Afterwards, the performance are quantified by means of some statistical indices, reported in Eqs. 41.42.

$$
\begin{gathered}
{ }_{n} \bar{\mu}=\frac{\sum_{i=1}^{100} R M S E_{i}}{100} \\
{ }_{n} \sigma_{\bar{\mu}}=\sqrt{\frac{\sum_{i=1}^{100}\left(\bar{\mu}-R M S E_{i}\right)^{2}}{100}}
\end{gathered}
$$

$R M S E_{i}$ is the root mean square error of the estimated variables computed at steady state for the $i^{\text {th }}$ simulation, ${ }_{n} \bar{\mu}$ and ${ }_{n} \sigma_{\bar{\mu}}$ are the mean and the standard deviation of RMSE, respectively, considering the filter of order $n$. Figure 12 provides a graphical reference for the different indices. $\bar{\mu}$ gives the mean accuracy of the filter, while $\sigma_{\bar{\mu}}$ quantifies the dispersion around the mean. If the standard deviation is high, the final accuracy strongly depends on the estimate of the initial condition and thus large initial errors may result in bad performance or even failure.

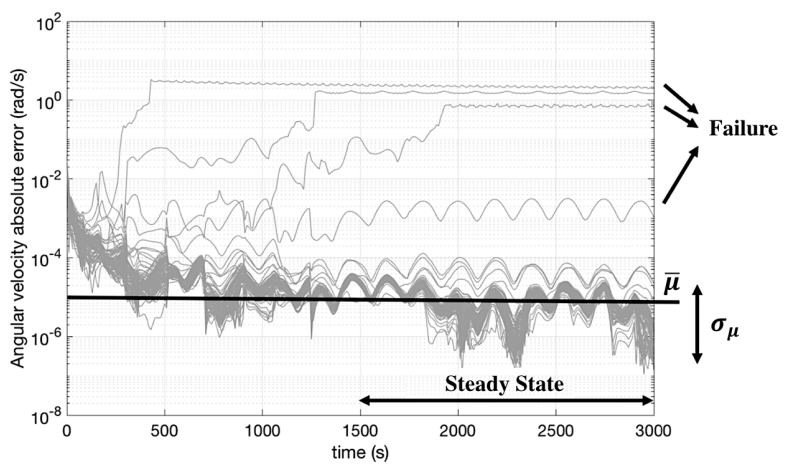

Figure 12: Graphical representation of the statistical indices.

In the following, the main outcomes of the simulations are discussed. All the detailed results of the numerical test campaign are reported in Di Lizia et al. ${ }^{26}$

\subsubsection{Translational dynamics filter}

As already pointed out, the translational dynamics is slow and almost linear since the two spacecrafts are very close on the same orbit, which is nearly circular. Therefore, 
high-order filters do not provide better performance w.r.t. a linear one, which is already capable of following the dynamic evolution. Indeed, both first and second order filters succeed in all the considered conditions of acquisition frequency and initial velocity uncertainty with the same estimation error at steady state, which is in the order of $10^{-2}$ $\mathrm{m}$ for the position and $10^{-7} \mathrm{~m} / \mathrm{s}$ for the velocity. As example, in Fig. 13 the absolute position and velocity errors considering a frequency of $3 \mathrm{~Hz}$ and $\sigma_{r_{r}, 0}=0.01 \mathrm{~m} / \mathrm{s}$ are reported.

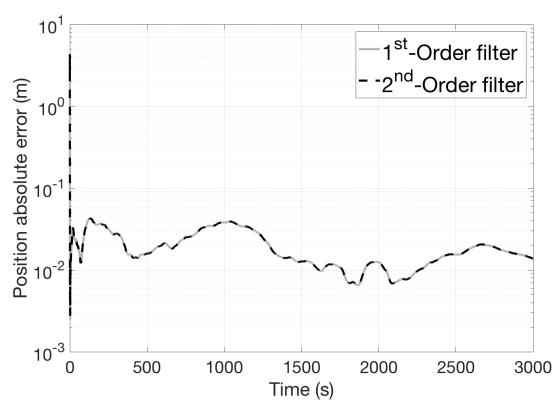

(a)

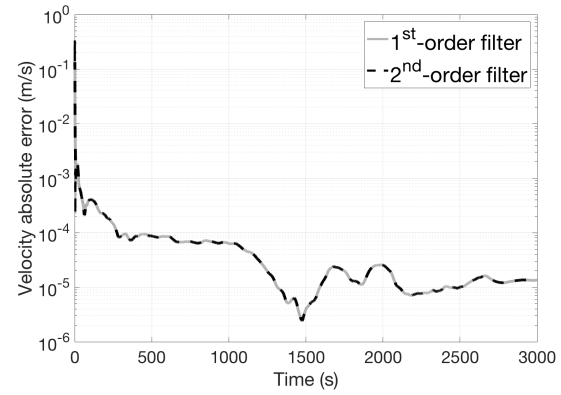

(b)

Figure 13: Position (a) and velocity (b) absolute error with a frequency of $3 \mathrm{~Hz}$ and $\sigma_{r_{r}, 0}=0.01 \mathrm{~m} / \mathrm{s}$.

\subsubsection{Rotational dynamics filter}

Regarding the rotational dynamics filter, the nonlinearities affect the estimation problem more significantly, especially in case of high uncertainties and low observability of the system. Focusing initially on the first case reported in Table 5, the performance of the first order filter, in terms of ${ }_{1} \bar{\mu}$ and ${ }_{1} \sigma_{\bar{\mu}}$, are shown in Tables 778 . Analogously, Tables 910 refer to the second order filter. These results are obtained taking into account only the converging solutions. For $\sigma_{\omega_{r}, 0}=0.1 \mathrm{rad} / \mathrm{s}$ and a frequency of $0.1 \mathrm{~Hz}$, neither of the two filters converge.

However, in order to compare the two filters and have a deeper insight into their 445 performance, the ratios $\frac{2 \bar{\mu}}{1 \bar{\mu}}$ and $\frac{2 \sigma_{\bar{\mu}}}{1 \sigma_{\bar{\mu}}}$ are computed and reported in Tables 11 12. The superscript reports the success percentage of the second order filter, while the subscript the success percentage of the first order filter.

On one hand, it can be observed that first and second order filters show the same 
performance for low uncertainties and high frequencies. However, moving to high uncertainties and low frequencies (shaded area), the second order filter starts outperforming the first order one. Indeed, even though ${ }_{1} \bar{\mu}$ is very similar to ${ }_{2} \bar{\mu},{ }_{1} \sigma_{\bar{\mu}}$ is significantly larger than ${ }_{2} \sigma_{\bar{\mu}}$, namely the first order filter features a higher dispersion of the steadystate estimation error (see Fig. 14). This means that, in case of large deviations from the true initial conditions, the first order filter performance deteriorates, leading to final estimates that are up to 1 order of magnitude worse than the ones of the second order filter (see Fig. 15).

Finally, the second order filter turns out to be also more robust in terms of failures. Indeed, in some cases, the first order filter is not able to deal with the nonlinearity and diverges, while the second order filter converges.

Table 7: ${ }_{1} \bar{\mu}$ in the sensitivity analysis to initial angular velocity uncertainty and acquisition frequency (nominal case).

\begin{tabular}{c|ccccc}
\hline \hline \multirow{2}{*}{$\begin{array}{c}\text { Freq. } \\
(\mathrm{Hz})\end{array}$} & 0.001 & 0.005 & 0.01 & 0.05 & 0.1 \\
\cline { 2 - 6 } 0.1 & $7.55 \mathrm{e}-04$ & $7.59 \mathrm{e}-04$ & $8.92 \mathrm{e}-04$ & $3.79 \mathrm{e}-03$ & - \\
0.4 & $1.16 \mathrm{e}-03$ & $1.16 \mathrm{e}-03$ & $1.16 \mathrm{e}-03$ & $1.29 \mathrm{e}-03$ & $1.47 \mathrm{e}-03$ \\
1 & $1.22 \mathrm{e}-03$ & $1.22 \mathrm{e}-03$ & $1.22 \mathrm{e}-03$ & $1.22 \mathrm{e}-03$ & $1.23 \mathrm{e}-03$ \\
3 & $5.35 \mathrm{e}-04$ & $5.35 \mathrm{e}-04$ & $5.35 \mathrm{e}-04$ & $5.35 \mathrm{e}-04$ & $5.35 \mathrm{e}-04$ \\
\hline
\end{tabular}

(a) Modified Rodrigues Parameters

\begin{tabular}{c|ccccc}
\hline \hline \multirow{2}{*}{$\begin{array}{c}\text { Freq. } \\
(\mathrm{Hz})\end{array}$} & 0.001 & 0.005 & 0.01 & 0.05 & 0.1 \\
\cline { 2 - 6 } 0.1 & $1.13 \mathrm{e}-05$ & $1.13 \mathrm{e}-05$ & $1.20 \mathrm{e}-05$ & $3.86 \mathrm{e}-05$ & - \\
0.4 & $3.91 \mathrm{e}-06$ & $3.91 \mathrm{e}-06$ & $3.91 \mathrm{e}-06$ & $4.45 \mathrm{e}-06$ & $4.81 \mathrm{e}-06$ \\
1 & $3.16 \mathrm{e}-06$ & $3.16 \mathrm{e}-06$ & $3.16 \mathrm{e}-06$ & $3.16 \mathrm{e}-06$ & $3.17 \mathrm{e}-06$ \\
3 & $3.06 \mathrm{e}-06$ & $3.07 \mathrm{e}-06$ & $3.06 \mathrm{e}-06$ & $3.07 \mathrm{e}-06$ & $3.07 \mathrm{e}-06$ \\
\hline
\end{tabular}

(b) Relative Angular Velocity (rad/s)

The analyses of the other examined cases provide similar results and stress the supe- 
Table 8: ${ }_{1} \sigma_{\bar{\mu}}$ in the sensitivity analysis to initial angular velocity uncertainty and acquisition frequency (nominal case).

\begin{tabular}{c|ccccc}
\hline \hline \multirow{2}{*}{$\begin{array}{c}\text { Freq. } \\
(\mathrm{Hz})\end{array}$} & 0.001 & 0.005 & 0.01 & 0.05 & 0.1 \\
\cline { 2 - 6 } & \multicolumn{5}{|c}{$\sigma_{\omega_{r}, 0}(\mathrm{rad} / \mathrm{s})$} \\
0.1 & $7.89 \mathrm{e}-06$ & $1.28 \mathrm{e}-05$ & $3.24 \mathrm{e}-04$ & $1.59 \mathrm{e}-03$ & - \\
0.4 & $5.26 \mathrm{e}-06$ & $3.40 \mathrm{e}-06$ & $3.92 \mathrm{e}-06$ & $3.79 \mathrm{e}-04$ & $3.38 \mathrm{e}-04$ \\
1 & $2.56 \mathrm{e}-06$ & $1.54 \mathrm{e}-06$ & $1.54 \mathrm{e}-06$ & $4.70 \mathrm{e}-06$ & $2.21 \mathrm{e}-05$ \\
3 & $1.13 \mathrm{e}-07$ & $7.03 \mathrm{e}-08$ & $7.19 \mathrm{e}-08$ & $7.25 \mathrm{e}-08$ & $1.05 \mathrm{e}-07$ \\
\hline
\end{tabular}

(a) Modified Rodrigues Parameters

\begin{tabular}{c|ccccc}
\hline \hline \multirow{2}{*}{$\begin{array}{c}\text { Freq. } \\
(\mathrm{Hz})\end{array}$} & 0.001 & 0.005 & 0.01 & 0.05 & 0.1 \\
\cline { 2 - 6 } 0.1 & $7.98 \mathrm{e}-08$ & $6.94 \mathrm{e}-08$ & $4.24 \mathrm{e}-06$ & $2.78 \mathrm{e}-05$ & - \\
0.4 & $2.95 \mathrm{e}-08$ & $1.61 \mathrm{e}-08$ & $1.89 \mathrm{e}-08$ & $1.82 \mathrm{e}-06$ & $1.95 \mathrm{e}-06$ \\
1 & $1.92 \mathrm{e}-09$ & $1.18 \mathrm{e}-09$ & $1.15 \mathrm{e}-09$ & $3.54 \mathrm{e}-09$ & $2.11 \mathrm{e}-08$ \\
3 & $4.40 \mathrm{e}-09$ & $2.56 \mathrm{e}-09$ & $2.34 \mathrm{e}-09$ & $2.67 \mathrm{e}-09$ & $3.30 \mathrm{e}-09$ \\
\hline
\end{tabular}

(b) Relative Angular Velocity (rad/s)
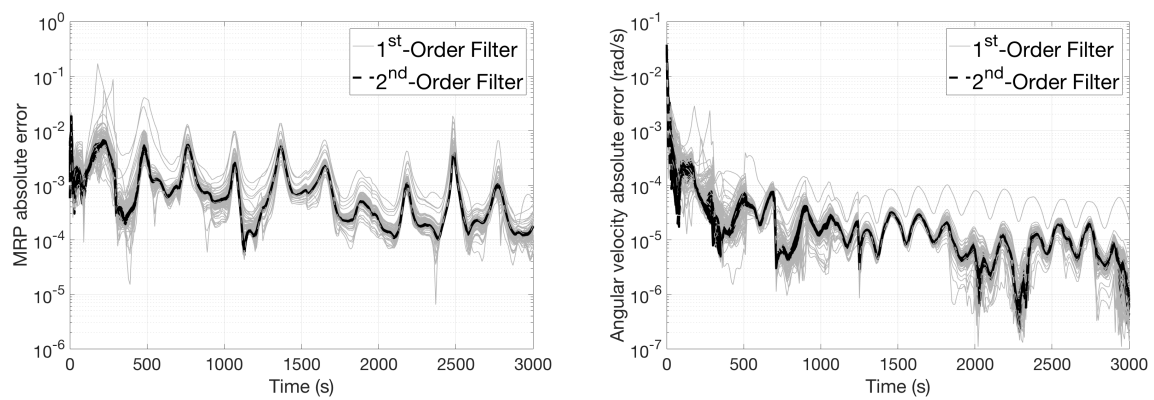

Figure 14: MRP (a) and angular velocity (b) absolute error with a frequency of $0.1 \mathrm{~Hz}$ and $\sigma_{\omega_{r}, 0}=0.01$ $\mathrm{rad} / \mathrm{s}$. 
Table 9: $2 \bar{\mu}$ in the sensitivity analysis to initial angular velocity uncertainty and acquisition frequency (nominal case).

\begin{tabular}{c|ccccc}
\hline \hline \multirow{2}{*}{$\begin{array}{c}\text { Freq. } \\
(\mathrm{Hz})\end{array}$} & 0.001 & 0.005 & 0.01 & 0.05 & 0.1 \\
\cline { 2 - 6 } & \multicolumn{5}{|c}{$\sigma_{\omega_{r}, 0}(\mathrm{rad} / \mathrm{s})$} \\
0.1 & $7.51 \mathrm{e}-04$ & $7.51 \mathrm{e}-04$ & $7.59 \mathrm{e}-04$ & $9.70 \mathrm{e}-04$ & - \\
0.4 & $1.16 \mathrm{e}-03$ & $1.16 \mathrm{e}-03$ & $1.16 \mathrm{e}-03$ & $1.16 \mathrm{e}-03$ & $1.18 \mathrm{e}-03$ \\
1 & $1.22 \mathrm{e}-03$ & $1.22 \mathrm{e}-03$ & $1.22 \mathrm{e}-03$ & $1.22 \mathrm{e}-03$ & $1.22 \mathrm{e}-03$ \\
3 & $5.35 \mathrm{e}-04$ & $5.35 \mathrm{e}-04$ & $5.35 \mathrm{e}-04$ & $5.35 \mathrm{e}-04$ & $5.35 \mathrm{e}-04$ \\
\hline
\end{tabular}

(a) Modified Rodrigues Parameters

\begin{tabular}{c|ccccc}
\hline \hline \multirow{2}{*}{$\begin{array}{c}\text { Freq. } \\
(\mathrm{Hz})\end{array}$} & 0.001 & 0.005 & 0.01 & 0.05 & 0.1 \\
\cline { 2 - 6 } & \multicolumn{5}{|c}{$\sigma_{\omega_{r}, 0}(\mathrm{rad} / \mathrm{s})$} \\
0.1 & $1.09 \mathrm{e}-05$ & $1.09 \mathrm{e}-05$ & $1.09 \mathrm{e}-05$ & $1.09 \mathrm{e}-05$ & - \\
0.4 & $4.61 \mathrm{e}-06$ & $4.61 \mathrm{e}-06$ & $4.61 \mathrm{e}-06$ & $4.61 \mathrm{e}-06$ & $4.61 \mathrm{e}-06$ \\
1 & $2.62 \mathrm{e}-06$ & $2.62 \mathrm{e}-06$ & $2.62 \mathrm{e}-06$ & $2.62 \mathrm{e}-06$ & $2.62 \mathrm{e}-06$ \\
3 & $1.48 \mathrm{e}-06$ & $1.48 \mathrm{e}-06$ & $1.48 \mathrm{e}-06$ & $1.48 \mathrm{e}-06$ & $1.48 \mathrm{e}-06$ \\
\hline
\end{tabular}

(b) Relative Angular Velocity ( $\mathrm{rad} / \mathrm{s})$
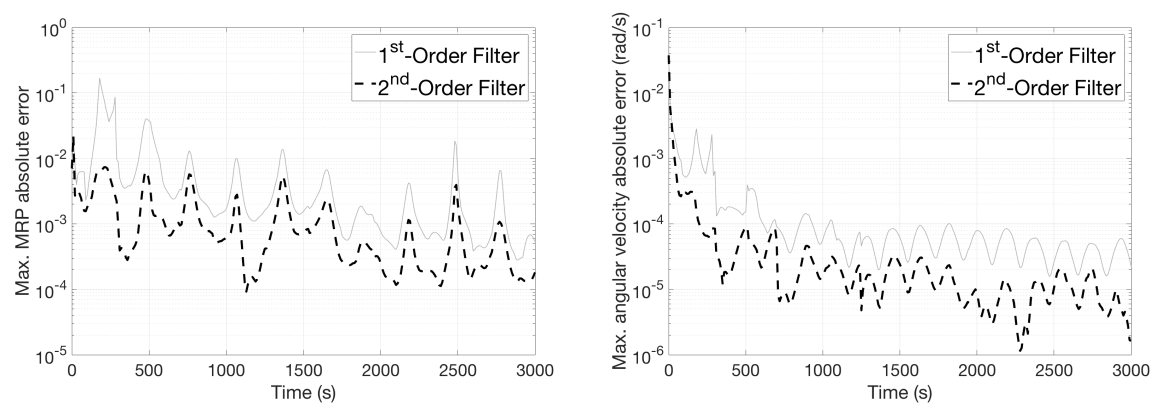

Figure 15: Maximum values of MRP (a) and angular velocity (b) absolute error with a frequency of $0.1 \mathrm{~Hz}$ and $\sigma_{\omega_{r}, 0}=0.01 \mathrm{rad} / \mathrm{s}$. 
Table 10: ${ }_{2} \sigma_{\bar{\mu}}$ in the sensitivity analysis to initial angular velocity uncertainty and acquisition frequency (nominal case).

\begin{tabular}{c|ccccc}
\hline \hline \multirow{2}{*}{$\begin{array}{c}\text { Freq. } \\
(\mathrm{Hz})\end{array}$} & 0.001 & 0.005 & 0.01 & 0.05 & 0.1 \\
\cline { 2 - 6 } & \multicolumn{5}{|c}{$\sigma_{\omega_{r}, 0}(\mathrm{rad} / \mathrm{s})$} \\
0.1 & $7.22 \mathrm{e}-06$ & $6.95 \mathrm{e}-06$ & $6.89 \mathrm{e}-06$ & $4.86 \mathrm{e}-04$ & - \\
0.4 & $5.24 \mathrm{e}-06$ & $3.38 \mathrm{e}-06$ & $3.78 \mathrm{e}-06$ & $3.25 \mathrm{e}-06$ & $3.93 \mathrm{e}-05$ \\
1 & $2.56 \mathrm{e}-06$ & $1.54 \mathrm{e}-06$ & $1.54 \mathrm{e}-06$ & $3.01 \mathrm{e}-06$ & $1.92 \mathrm{e}-06$ \\
3 & $1.14 \mathrm{e}-07$ & $7.08 \mathrm{e}-08$ & $7.23 \mathrm{e}-08$ & $7.25 \mathrm{e}-08$ & $9.68 \mathrm{e}-08$ \\
\hline
\end{tabular}

(a) Modified Rodrigues Parameters

\begin{tabular}{c|ccccc}
\hline \hline \multirow{2}{*}{$\begin{array}{c}\text { Freq. } \\
(\mathrm{Hz})\end{array}$} & 0.001 & 0.005 & 0.01 & 0.05 & 0.1 \\
\cline { 2 - 6 } & \multicolumn{5}{|c}{$\sigma_{\omega_{r}, 0}(\mathrm{rad} / \mathrm{s})$} \\
0.1 & $7.06 \mathrm{e}-08$ & $5.51 \mathrm{e}-08$ & $9.31 \mathrm{e}-08$ & $4.28 \mathrm{e}-06$ & - \\
0.4 & $2.93 \mathrm{e}-08$ & $1.60 \mathrm{e}-08$ & $1.85 \mathrm{e}-08$ & $2.32 \mathrm{e}-08$ & $1.52 \mathrm{e}-07$ \\
1 & $1.90 \mathrm{e}-09$ & $1.16 \mathrm{e}-09$ & $1.14 \mathrm{e}-09$ & $2.26 \mathrm{e}-09$ & $1.34 \mathrm{e}-09$ \\
3 & $4.41 \mathrm{e}-09$ & $2.56 \mathrm{e}-09$ & $2.35 \mathrm{e}-09$ & $2.66 \mathrm{e}-09$ & $3.15 \mathrm{e}-09$ \\
\hline
\end{tabular}

(b) Relative Angular Velocity (rad/s) 
Table 11: $\frac{2 \bar{\mu}}{1 \bar{\mu}}$ in the sensitivity analysis to initial angular velocity uncertainty and acquisition frequency (nominal case).

\begin{tabular}{c|ccccc}
\hline \hline \multirow{2}{*}{$\begin{array}{c}\text { Freq. } \\
(\mathrm{Hz})\end{array}$} & 0.001 & 0.005 & 0.01 & 0.05 & 0.1 \\
\cline { 2 - 6 } & \multicolumn{5}{|c}{$\sigma_{\omega_{r}, 0}(\mathrm{rad} / \mathrm{s})$} \\
0.1 & $0.994_{100}^{100}$ & $0.993_{100}^{100}$ & $0.850_{93}^{100}$ & $0.255_{11}^{50}$ & $-_{0}^{0}$ \\
0.4 & $0.996_{100}^{100}$ & $0.997_{100}^{100}$ & $0.997_{100}^{100}$ & $0.902_{88}^{100}$ & $0.800_{96}^{99}$ \\
1 & $0.988_{100}^{100}$ & $0.989_{100}^{100}$ & $0.989_{100}^{100}$ & $0.991_{100}^{100}$ & $0.996_{100}^{100}$ \\
3 & $1.000_{100}^{100}$ & $1.000_{100}^{100}$ & $1.000_{100}^{100}$ & $1.000_{100}^{100}$ & $1.000_{100}^{100}$ \\
\hline
\end{tabular}

(a) Modified Rodrigues Parameters

\begin{tabular}{c|ccccc}
\hline \hline \multirow{2}{*}{$\begin{array}{c}\text { Freq. } \\
(\mathrm{Hz})\end{array}$} & 0.001 & 0.005 & 0.01 & 0.05 & 0.1 \\
\cline { 2 - 6 } & \multicolumn{5}{|c}{$\sigma_{\omega_{r}, 0}(\mathrm{rad} / \mathrm{s})$} \\
0.1 & $0.998_{100}^{100}$ & $0.998_{100}^{100}$ & $0.941_{93}^{100}$ & $0.334_{11}^{50}$ & $-_{0}^{0}$ \\
0.4 & $0.999_{100}^{100}$ & $0.999_{100}^{100}$ & $0.999_{100}^{100}$ & $0.881_{88}^{100}$ & $0.815_{96}^{99}$ \\
1 & $0.999_{100}^{100}$ & $0.999_{100}^{100}$ & $0.999_{100}^{100}$ & $0.999_{100}^{100}$ & $0.997_{100}^{100}$ \\
3 & $1.000_{100}^{100}$ & $1.000_{100}^{100}$ & $1.000_{100}^{100}$ & $1.000_{100}^{100}$ & $1.000_{100}^{100}$ \\
\hline
\end{tabular}

(b) Relative Angular Velocity (rad/s) 
Table 12: $\frac{2 \sigma_{\bar{\mu}}}{1 \sigma_{\bar{\mu}}}$ in the sensitivity analysis to initial angular velocity uncertainty and acquisition frequency (nominal case).

\begin{tabular}{c|ccccc}
\hline \hline \multirow{2}{*}{$\begin{array}{c}\text { Freq. } \\
(\mathrm{Hz})\end{array}$} & 0.001 & 0.005 & 0.01 & 0.05 & 0.1 \\
\cline { 2 - 6 } & \multicolumn{5}{|c}{$\sigma_{\omega_{r}, 0}(\mathrm{rad} / \mathrm{s})$} \\
0.1 & $0.914_{100}^{100}$ & $0.543_{100}^{100}$ & $0.021_{93}^{100}$ & $0.305_{11}^{50}$ & $-_{0}^{0}$ \\
0.4 & $0.998_{100}^{100}$ & $0.995_{100}^{100}$ & $0.965_{100}^{100}$ & $0.008_{88}^{100}$ & $0.116_{96}^{99}$ \\
1 & $0.999_{100}^{100}$ & $0.999_{100}^{100}$ & $0.997_{100}^{100}$ & $0.641_{100}^{100}$ & $0.086_{100}^{100}$ \\
3 & $1.000_{100}^{100}$ & $1.000_{100}^{100}$ & $1.000_{100}^{100}$ & $0.999_{100}^{100}$ & $0.919_{100}^{100}$ \\
\hline
\end{tabular}

(a) Modified Rodrigues Parameters

\begin{tabular}{c|ccccc}
\hline \hline \multirow{2}{*}{$\begin{array}{c}\text { Freq. } \\
(\mathrm{Hz})\end{array}$} & 0.001 & 0.005 & 0.01 & 0.05 & 0.1 \\
\cline { 2 - 6 } & \multicolumn{5}{|c}{$\sigma_{\omega_{r}, 0}(\mathrm{rad} / \mathrm{s})$} \\
0.1 & $0.884_{100}^{100}$ & $0.794_{100}^{100}$ & $0.022_{93}^{100}$ & $0.157_{11}^{50}$ & $-_{0}^{0}$ \\
0.4 & $0.994_{100}^{100}$ & $0.994_{100}^{100}$ & $0.998_{100}^{100}$ & $0.012_{88}^{100}$ & $0.078_{96}^{99}$ \\
1 & $0.989_{100}^{100}$ & $0.986_{100}^{100}$ & $0.989_{100}^{100}$ & $0.638_{100}^{100}$ & $0.633_{100}^{100}$ \\
3 & $1.000_{100}^{100}$ & $1.000_{100}^{100}$ & $1.000_{100}^{100}$ & $0.998_{100}^{100}$ & $0.955_{100}^{100}$ \\
\hline
\end{tabular}

(b) Relative Angular Velocity (rad/s) 
riority of the second order filter for high uncertainties and low acquisition frequencies. The same result is obtained for the different levels of measurement noise as reported in Tables 13 and 14 In fact, higher uncertainties, either in dynamics knowledge or in the measurements (Tables 5 6 , respectively), cause an increase of the final error dispersion of the linear filter and thus a worse performance deterioration with respect to the nonlinear one, leading to errors up to two orders of magnitude larger. On the other hand, when the initial angular rate is increased (Table 5), the area in which the second order filter is more robust becomes larger, for equal uncertainty and frequency domain. This case is similar to reducing the acquisition frequency and thus the observability of the system, increasing nonlinearity effects.

Table 13: $\frac{2 \bar{\mu}}{1 \bar{\mu}}$ in the sensitivity analysis to the levels of measurement noise from Table 6

\begin{tabular}{c|ccc}
\hline \hline \multirow{2}{*}{$\begin{array}{c}\text { Freq. } \\
(\mathrm{Hz})\end{array}$} & 1 & 2 & 4 \\
\cline { 2 - 4 } 0.4 & $0.997_{100}^{100}$ & $0.960_{91}^{99}$ & $0.994_{75}^{98}$ \\
3 & $1.000_{100}^{100}$ & $1.000_{100}^{100}$ & $0.999_{99}^{99}$ \\
\hline
\end{tabular}

(a) Modified Rodrigues Parameters

\begin{tabular}{c|ccc}
\hline \hline \multirow{2}{*}{$\begin{array}{c}\text { Freq. } \\
(\mathrm{Hz})\end{array}$} & \multicolumn{3}{|c}{$G$} \\
\cline { 2 - 4 } 0.4 & $0.999_{100}^{100}$ & $0.973_{91}^{99}$ & $1.004_{75}^{98}$ \\
3 & $1.000_{100}^{100}$ & $1.001_{100}^{100}$ & $1.000_{99}^{99}$ \\
\hline
\end{tabular}

(b) Relative Angular Velocity ( $\mathrm{rad} / \mathrm{s})$

\subsection{Computational time on the BeagleBone Black}

This section addresses the assessment of the required computational effort of the DAbased HNEKF on the BeagleBone Black (BBB) Single Board Computer, based on an ARMv7 processor (Cortex A8) @ 1GHz with 512Mb of RAM. The BBB is deemed to be representative of the limited computational capability available on onboard space processors. The filter is entirely compiled out of $\mathrm{C} 11$ code directly on the target ARM 
Table 14: $\frac{2 \sigma_{\bar{\mu}}}{1 \sigma_{\bar{\mu}}}$ in the sensitivity analysis to the levels of measurement noise from Table 6

\begin{tabular}{c|ccc}
\hline \hline \multirow{2}{*}{$\begin{array}{c}\text { Freq. } \\
(\mathrm{Hz})\end{array}$} & \multicolumn{3}{|c}{$G$} \\
\cline { 2 - 4 } 0.4 & $0.965_{100}^{100}$ & $0.042_{91}^{99}$ & $0.037_{75}^{98}$ \\
3 & $1.000_{100}^{100}$ & $1.020_{100}^{100}$ & $0.887_{99}^{99}$ \\
\hline
\end{tabular}

(a) Modified Rodrigues Parameters

\begin{tabular}{c|ccc}
\hline \hline \multirow{2}{*}{$\begin{array}{c}\text { Freq. } \\
(\mathrm{Hz})\end{array}$} & 1 & 2 & 4 \\
\cline { 2 - 4 } 0.4 & $0.998_{100}^{100}$ & $0.033_{91}^{99}$ & $0.088_{75}^{98}$ \\
3 & $1.000_{100}^{100}$ & $1.002_{100}^{100}$ & $1.023_{99}^{99}$ \\
\hline
\end{tabular}

(b) Relative Angular Velocity (rad/s)

platform, which is running a tailored Linux 4.9 kernel and proper GCC compiler.

In order to asses the feasibility of the developed filter on the embedded hardware, a Real-Time Operative System (RTOS) should have been employed, allowing the real time scheduling of the filter task at the desired frequency. However, the filter does not really acquire measurements since those are generated in advance by the dynamics simulator. Therefore, an accurate real time scheduling is not strictly required. Indeed, the computational time required by each step of the filter can be measured and compared to the time step at the desired frequency, checking that it is smaller.

To this aim, the duty cycle concept is introduced. The duty cycle represent the fraction of the available sampling time which is used by the filter task, as shown in Fig. 16. Therefore, given the execution time $t_{\text {exec }}$ and defining the sampling time as:

$$
t_{a}=\frac{1}{f_{a}}
$$

the duty cycle is:

$$
D C=\frac{t_{\text {exec }}}{t_{a}}
$$




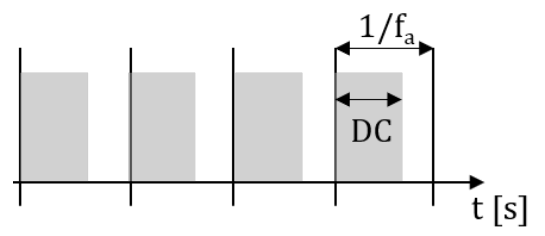

Figure 16: Duty cycle concept

The analysis is carried out considering different sampling frequency and processor clock frequency on the BBB. More specifically, the sampling frequencies are $f_{a}=$ $[0.1,0.4,1,3] \mathrm{Hz}$ while the clock frequencies are $c l k=\left[100^{2} 275,720,1000\right] \mathrm{MHz}$ . First and second order filters are executed considering both the translational and rotational dynamics. The results of the execution time and duty cycle are reported in Table 15

Table 15: Filter execution on BBB

\begin{tabular}{|c|c|c|c|c|c|c|c|c|c|}
\hline \multicolumn{5}{|c|}{ Execution Time [s] - Order 1} & \multicolumn{5}{|c|}{ Duty Cycle [\%] - Order 1} \\
\hline $\mathrm{f}_{a} \backslash \mathrm{clk}$ & 100 & 275 & 720 & 1000 & $\mathrm{f}_{a} \backslash \mathrm{clk}$ & 100 & 275 & 720 & 1000 \\
\hline 0.1 & 0.381 & 0.303 & 0.110 & 0.006 & 0.1 & $3.8 \%$ & $1.2 \%$ & $0.1 \%$ & $0.0 \%$ \\
\hline 0.4 & 0.115 & 0.091 & 0.035 & 0.004 & 0.4 & $4.6 \%$ & $0.4 \%$ & $0.0 \%$ & $0.0 \%$ \\
\hline 1 & 0.062 & 0.049 & 0.018 & 0.002 & 1 & $6.2 \%$ & $0.3 \%$ & $0.0 \%$ & $0.0 \%$ \\
\hline 3 & 0.026 & 0.021 & 0.008 & 0.001 & 3 & $7.8 \%$ & $0.2 \%$ & $0.0 \%$ & $0.0 \%$ \\
\hline \multicolumn{5}{|c|}{ Execution Time [s] - Order 2} & \multicolumn{5}{|c|}{ Duty Cycle [\%] - Order 2} \\
\hline $\mathrm{f}_{a} \backslash \mathrm{clk}$ & 100 & 275 & 720 & 1000 & $\mathrm{f}_{a} \backslash \mathrm{clk}$ & 100 & 275 & 720 & 1000 \\
\hline 0.1 & 1.342 & 1.072 & 0.413 & 0.043 & 0.1 & $13.4 \%$ & $14.4 \%$ & $5.9 \%$ & $0.3 \%$ \\
\hline 0.4 & 0.426 & 0.339 & 0.129 & 0.014 & 0.4 & $17.0 \%$ & $5.8 \%$ & $0.7 \%$ & $0.0 \%$ \\
\hline 1 & 0.25 & 0.199 & 0.077 & 0.011 & 1 & $25.0 \%$ & $5.0 \%$ & $0.4 \%$ & $0.0 \%$ \\
\hline 3 & 0.124 & 0.099 & 0.039 & 0.007 & 3 & $37.2 \%$ & $3.7 \%$ & $0.1 \%$ & $0.0 \%$ \\
\hline
\end{tabular}

${ }^{2}$ Interpolated 
cycle remains always well below the 50\%, thus allowing for the filtering and also other necessary tasks. Not surprisingly, the duty cycle increases when reducing the clock frequency as the processor is capable of executing less operation per seconds. Moreover, at constant clock frequency, the duty cycle reduces when reducing the sampling frequency, demonstrating that the longer propagation time span, needed for computing the expectations, is not highly influencing the overall computational time. Concentrating on the lower clock frequency, it is possible to see how the second order filter is more feasible at lower sampling frequencies, that are the cases in which this filter outperforms the first order version.

\section{Conclusion}

The work investigated the possibility and assessed the advantages of onboard $6 \mathrm{DoF}$ state estimation using DA techniques. The problem of real-time relative pose estimation during proximity operations has been considered as target application, using the e.deorbit mission with the target Envisat as reference scenario. To attain this goal, a DA-based HNEKF has been developed and implemented on a BeagleBone Black platform, which is deemed to be representative of the limited computational performance available on current onboard space processors. An analysis on the effects of including high order terms in the EKF implementation has been carried out and has highlighted that using orders greater than two does not improve the accuracy of the estimation. This result has been proven to be related to the hypothesis of the filter that all random distributions are Gaussian and, thus, completely described by their mean and covariance. In addition, through an extensive sensitivity analysis, it has been shown that the second order filter tends to outperform the classical first order counterpart either for relatively large initial errors and uncertainties, or for relatively low acquisition frequencies. Nevertheless, the second order filter outperforms its first order version in terms of final error dispersion and robustness to failure. Finally, the tests have demonstrated that the second order filter can be run on an ARM processor @ $100 \mathrm{MHz}$ for the target application. 


\section{Acknowledgments}

This work has been carried out in collaboration with the Advanced Concepts Team under the Ariadna study "Assessment of onboard DA state estimation for spacecraft relative navigation" (Contract No. IPL-PTE/LF/as/517.2016).

\section{References}

\section{References}

[1] O. Montenbruck, E. Gill, Satellite orbits: models, methods and applications, Springer Science \& Business Media, 2012.

[2] S. Julier, J. Uhlmann, H. Durrant-Whyte, A new approach for filtering nonlinear systems, in: Proceedings of the IEEE, Vol. 3, 1995, pp. 1628-1632.

[3] S. J. Julier, J. K. Uhlmann, Unscented filtering and nonlinear estimation, in: Proceedings of the IEEE, Vol. 92, IEEE, 2004, pp. 401-422.

[4] R. Park, D. Scheeres, Nonlinear mapping of gaussian statistics: theory and applications to spacecraft trajectory design, Journal of Guidance Control and Dynamics 29 (6) (2006) 1367-1375.

[5] R. Park, D. Scheeres, Nonlinear semi-analytic methods for trajectory estimation, Journal of Guidance Control and Dynamics 30 (6) (2007) 1668-1676.

[6] M. Valli, R. Armellin, P. Di Lizia, M. Lavagna, Nonlinear filtering methods for spacecraft navigation based on differential algebra, Acta Astronautica 94 (2014) $363-374$

[7] M. Berz, The method of power series tracking for the mathematical description of beam dynamics, Nuclear Instruments and Methods in Physics Research Section A: Accelerators, Spectrometers, Detectors and Associated Equipment 258 (3) (1987) 431-436.

[8] M. Berz, Modern Map Methods in Particle Beam Physics, Academic Press, 1999. 
[9] R. Armellin, P. Di Lizia, F. Bernelli-Zazzera, M. Berz, Asteroid close encounters characterization using differential algebra: the case of apophis, Celestial Mechanics and Dynamical Astronomy 107 (2010) 451-470.

[10] A. Morselli, R. Armellin, P. Di Lizia, F. Bernelli-Zazzera, A high order method for orbital conjunctions analysis: sensitivity to initial uncertainties, Advances in Space Research 55 (2015) 311-333.

[11] M. Rasotto, A. Morselli, A. Wittig, M. Massari, P. Di Lizia, R. Armellin, C. Valles, G. Ortega, Differential algebra space toolbox for nonlinear uncertainty propagation in space dynamics, in: Proceedings of the 6th International Conference on Astrodynamics Tools and Techniques, 2016.

[12] M. Massari, P. Di Lizia, M. Rasotto, Nonlinear uncertainty propagation in astrodynamics using differential algebra and graphics processing units, Journal of Aerospace Information Systems 14 (9) (2017) 493-503.

[13] R. Biesbroek, T. Soares, J. Husing, L. Innocenti, The e. deorbit cdf study: A design study for the safe removal of a large space debris, in: 64th International Astronautical Congress (IAC), Beijing, 2013.

[14] J. Kelsey, J. Byrne, M. Cosgrove, S. Seereeram, R. Mehra, Vision-based relative pose estimation for autonomous rendezvous and docking, in: Proceedings of IEEE Aerospace Conference, 2006, pp. 1-20.

[15] C. Liu, H. Weiduo, Relative pose estimation for cylinder-shaped spacecraft using single image, IEEE Transactions on Aerospace and Electronic Systems 50 (2014) $570 \quad 3036-3056$.

[16] R. Biesbroek, The e.deorbit esa cdf study, in: Proceedings of the SPIE, Vol. 5088, 2003, pp. 20-30.

[17] M. Själander, S. Habinc, J. Gaisler, Leon4: Fourth generation of the leon processor, in: Proceedings of Data Systems in Aerospace. 
[18] M. Massari, P. Di Lizia, F. Cavenago, A. Wittig, Differential algebra software library with automatic code generation for space embedded applications, AIAA SciTech Forum, American Institute of Aeronautics and Astronautics, 2018. doi : $10.2514 / 6.2018-0398$

[19] L. Isserlis, On a formula for the product-moment coefficient of any order of a normal frequency distribution in any number of variables, Biometrika 12 (1/2) (1918) 134-139.

[20] G. Casella, R. L. Berger, Statistical inference, Vol. 2, Duxbury Pacific Grove, CA, 2002.

[21] M. Valli, R. Armellin, P. Di Lizia, M. Lavagna, Nonlinear mapping of uncertainties in celestial mechanics, Journal of Guidance Control and Dynamics 36 (2013) $48-63$.

[22] J. L. Junkins, H. Schaub, Analytical mechanics of space systems, American Institute of Aeronautics and Astronautics, 2009.

[23] M. Massari, M. Zamaro, Application of sdre technique to orbital and attitude control of spacecraft formation flying, Acta Astronautica 94 (1) (2014) 409-420.

[24] S. Marandi, V. Modi, A preferred coordinate system and the associated orientation representation in attitude dynamics, Acta Astronautica 15 (11) (1987) 833-843.

[25] J. Gil-Fernández, T. Prieto-Llanos, R. Panzeca, Autonomous gnc algorithms for neo impactor missions, in: Proceedings of the 20th International Symposium on Space Flight Dynamics, 2007, pp. 1-14.

[26] P. Di Lizia, M. Massari, F. Cavenago, Assessment of onboard da state estimation for spacecraft relative navigation, Final Report IPL-PTE/LF/as/517.2016, European Space Agency (2017).

a URL http://wWW.esa.int/gsp/ACT/doc/ARI/ARI\%20Study\%20Report/ 600 ACT-RPT-MAD-ARI-16-5201-Assessment_of_onboard_DA_state_ estimation_for_spacecraft_relative_navigation.pdf 